\title{
Predicting of the Seismogram and Accelerogram of Strong Motions of the Soil for an Earthquake Model Considered as an Instantaneous Rupture of the Earth's Surface
}

\author{
Eduard Khachiyan \\ "Building Mechanics" Department, National University of Architecture and Construction of Armenia, Yerevan, Republic of Armenia \\ Email address: \\ edkhach@sci.am
}

To cite this article:

Eduard Khachiyan. Predicting of the Seismogram and Accelerogram of Strong Motions of the Soil for an Earthquake Model Considered as an Instantaneous Rupture of the Earth's Surface. Earth Sciences. Vol. 7, No. 4, 2018, pp. 183-201. doi: 10.11648/j.earth.20180704.16

Received: May 4, 2018; Accepted: July 12, 2018; Published: August 30, 2018

\begin{abstract}
Problems of the prediction of displacement and acceleration values for strong soil displacements are considered for the case where an earthquake is regarded as an instantaneous mechanical rupture of the Earth's surface. We have attempted to develop, based on recent concepts of earthquake generation process, simplified theoretical methods for the quantitative prediction of soil displacement parameters during strong earthquakes. As an illustrative example, we consider an earthquake originating as a consequence of relative displacements of suddenly ruptured blocks in a horizontal direction with a given initial velocity. An empirical relationship between soil particle motion velocity near the rupture and at a certain distance from it, on one hand, and the earthquake magnitude, on the other hand, was established. It is assumed that the impact of inertial motions of a deep soil stratum on the inertial motions of upper subsurface soil stratum at instantaneous break of a medium can be neglected. By solving a wave problem for a multilayer near surface stratum, analytical relations were developed for a soil seismogram and accelerogram on the surface depending on the physical-mechanical and dynamic characteristics of the soil at all layers of the stratum; attenuation coefficients of mechanical soil vibrations; the distance to the rupture; and the magnitude of the predicted earthquake. The results obtained enable us to determine the maximum displacement and acceleration values of the soil, taking into account local soil conditions and their variations over time, as well as the values of the predominant vibration periods in the soil. The method was applied for solid and loose soil basements.
\end{abstract}

Keywords: Earthquake, Instantaneous Rupture, Initial Velocity, Multilayer Stratum, Wave Problem, Predominant Periods, Seismogram, Accelerogram

\section{Introduction}

One of the main objectives of earthquake engineering (engineering seismology) is to predict patterns of soil vibrations (a construction site, for example) and their amplitude-frequency characteristics depending on local geological conditions during strong earthquakes. Reliable data on the character of earthquake induced soil vibrations in the soil can be obtained only by records made during a real earthquake. Owing to the large number of records of past earthquakes for the area of study, characteristic types of earthquakes can be distinguished. But, the number of such records is still insufficient for full statistical processing. This concerns both the entire territory of the former USSR and the Republic of Armenia in particular.
The term of "vibration record of the soil" usually refers to the record of soil motion (seismogram) and acceleration record of the soil motion (accelerogram). Soil displacement induced by strong earthquakes in the epicentral area can be so essential that accurate instrumental reproduction becomes technically infeasible. Soil displacement can be recorded with a high degree of reliability at epicenters of mainly weak earthquakes and at a large distance from the epicenter during strong earthquakes. Nowadays, all seismically active regions have been characterized by a large number of such records. In both cases, these records (seismograms), in terms of their application to the evaluation of the seismic resistance of buildings, are of little real interest, since the stress level in structural units of a building construction induced by weak earthquakes, relative to maximum permissible level, is 
significantly low.

It should be emphasized that there are no problems in finding acceleration records of soil motion. At present, networks of stations equipped with high-performance three-component accelerographs for recording strong soil displacements and accelerations of the soil motion both in a strong earthquake epicentral area and at a considerably great distance from its epicenter, are in many seismically active regions. During an earthquake, along with permanent (own weight) and temporary (the total weight of a building construction, snow, wind pressure) loads, so-called seismic loads affect buildings and constructions. Actually, there are no loads (forces) in the usual sense of the word. When the soil moves beneath the structure, the structure itself falls behind the motion of the soil, as a result of inertia, and bends. This is like the effect of horizontal forces on a building perpendicular to its axis. The values of these forces (alternating inertial loads) are primarily determined by the alternating soil acceleration magnitude, as well as weight and stiffness of a structure. All loads, except for seismic loads, create a direct physical impact on an object and have constant directions.

(a)

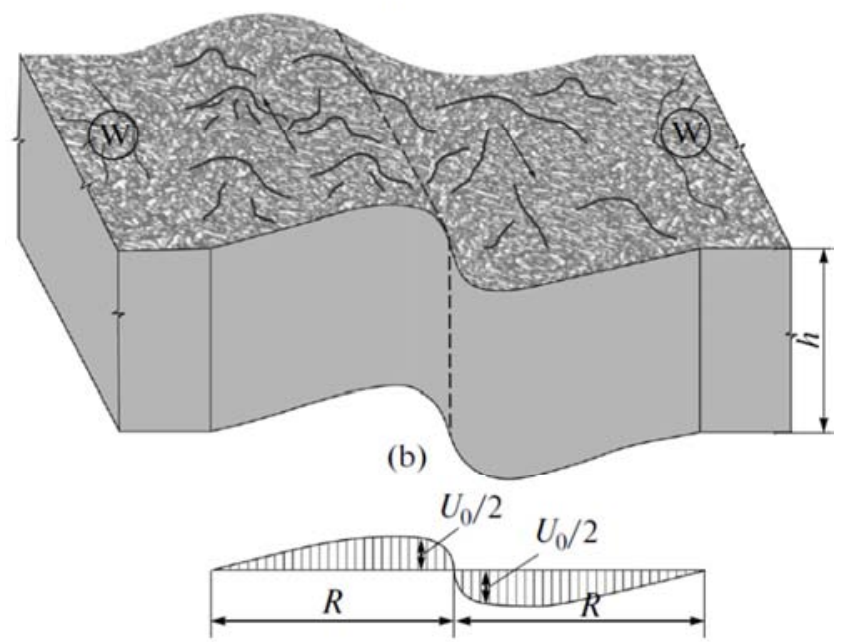

Figure 1. Schematic representation of the slow (long-term) deformation of a medium during a period of earthquake preparation: (a) strain state of the medium before faulting; (b) the medium displacement distribution in the direction perpendicular to the rupture before an earthquake, $h-a$ depth of a future rupture; $U_{d} / 2$ - static deformation of blocks at rupture; $R$ - the extent of the deformation field in the perpendicular direction to the rupture; $W$-areas taken as nondeformed by a future earthquake due to the relatively small strain values. The arrows demonstrate the directions of slow movements of blocks; dotted line is future rupture line.

Dynamic seismic load is only valid during an earthquake. Hence, parameters characterizing the level of seismic hazard of the given area are determined primarily by horizontal (vertical, rotational) soil-displacement acceleration and its variation over time. However, as noted above, the number of such parameters for most seismically active areas are insufficient.

There are numerous empirical formulas allowing one to determine only maximum soil acceleration values, depending on the magnitude, focal depth, and distance to an observation site. When designing responsible constructions, engineers often synthesize artificial accelerograms using real accelerograms recorded in an area with similar geotechnical conditions. The acceleration ordinate of a real accelerogram is usually reduced or increased. In addition, there are computer programs that allow one to create an artificial accelerogram of a strong earthquake for the observation site based on records of real seismograms and accelerograms of weak earthquakes. When solving problems of the seismic stability of complex and extended structures, designers need not only accelerograms of a strong earthquake, but also seismograms. All of the above shows the relevance of studies that allow one to develop approaches to creating artificial seismograms and accelerograms of strong soil displacement, adequately reflecting the properties of real seismic events recorded.

This article discusses a particular approach to predicting the displacement and acceleration values of strong soil motions considering an earthquake as an instantaneous break of the Earth's surface. As an example, we consider an earthquake induced by displacement of ruptured blocks relative to each other in a horizontal direction (strike-slip fault). In this case, the initial velocity of soil particles near the rupture after an earthquake is the main parameter characterizing the earthquake magnitude. Based on theoretical studies on determining the maximum velocity and acceleration values of the soil near a point source J. Brune, study results given in works by G. Reed, Lomnica, S. K. Singh, L. Esteva, K. Kasahara, D. Wells, and K. Coppersmith, as well as our own data, we established an empirical relationship between the maximum velocity of soil particles near the rupture and at any distance from it and the predicted earthquake magnitude.

\section{Initial Parameters of the Task}

\subsection{Initial Velocity of Displacement the Blocks}

According to modern concepts, an earthquake results from a mechanical break or rupture of the medium due to the slow motion of two contacted geological units with uneven boundaries in opposite directions. Before an earthquake, continuously increasing mechanical stresses, caused by the slow deformation of rocks, surrounding a future focus, appear in an area of the medium (Figure 1a). The strongest static deformations $U_{0} / 2$ will be recorded in the vicinity of a future rupture. At some distance $R$ perpendicular to direction of the rupture (Figure 1b), the magnitudes of these deformations can be regarded negligible compared with $U_{0} / 2$ near a proposed rupture [1, 6, 12, 15]. According to Reed and Brun [1, 15]: "The only way for the abovementioned stresses to be discharged is relative soil displacement on the opposite ends of a fault zone and at a distance from it" [15]; "The static displacement field, which determines the double dipole, without moment decreases with a distance proportionally to $\Delta^{-2}$ [1] (where $\Delta$ is a distance from a point source (focus) to an observation point).

Methods for determining distances $R$ and their quantitative values for 44 strong earthquakes are given in $[11,13]$. In 
particular, $R$ values can be determined by a mean (over the length of a rupture) displacement value $\bar{U}_{0}$ [22], resulting

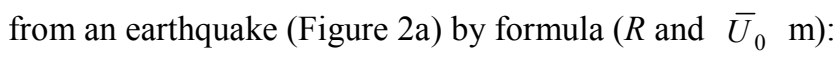

$$
R=\left(5 \cdot \bar{U}_{0}+15\right) \times 10^{3}
$$

(a)

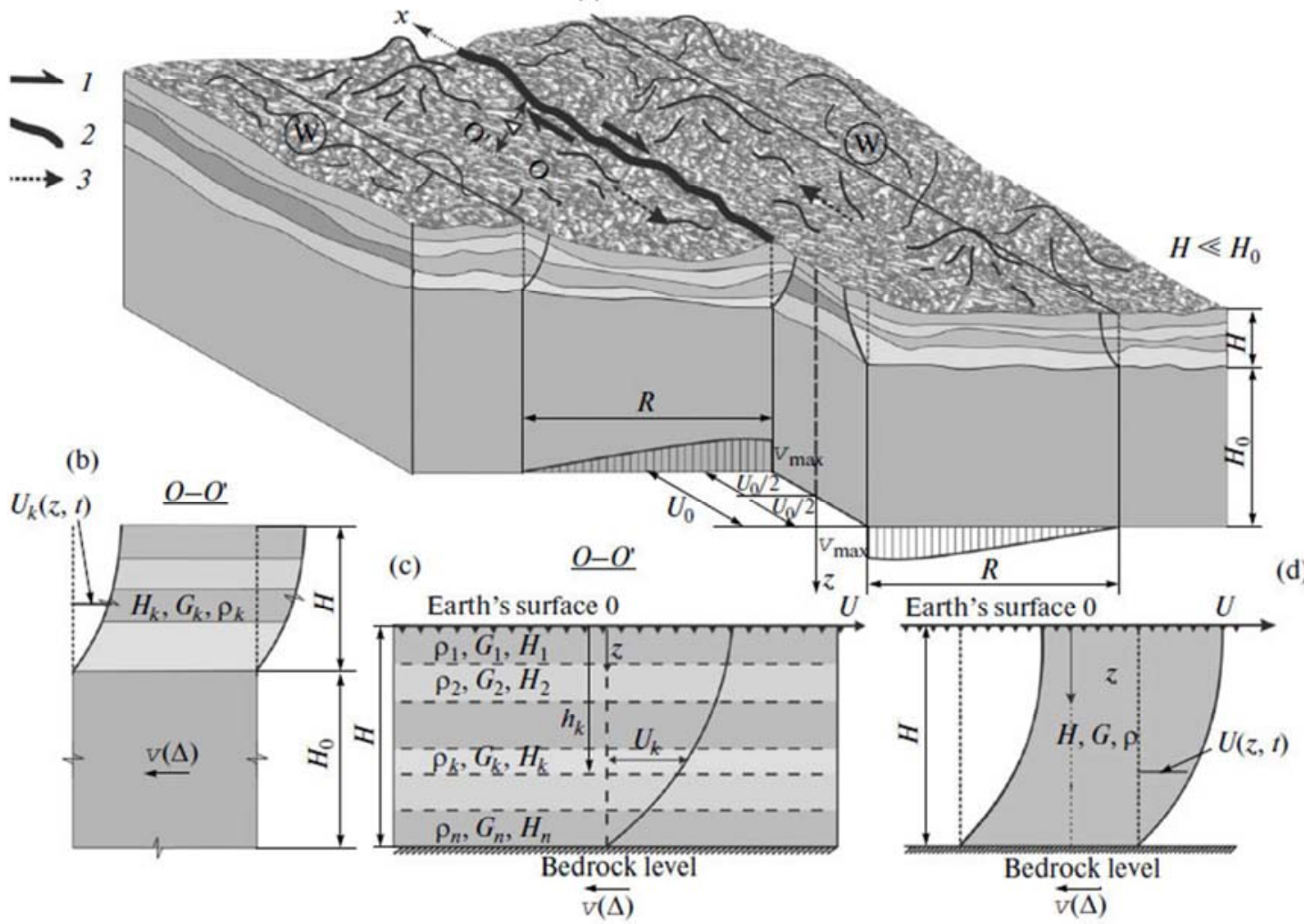

Figure 2. Schematic representation of the medium after earthquake rupture: (a) scheme of the rupture development and the mechanical model of the upper strata: (b) acceptable scheme of horizontal deformation of the vertical section along the $O-O^{\prime}$ line; (c) the scheme of upper strata in the form of an inhomogeneous surface strata; (d) horizontal deformation of a homogeneous surface layer. $H$ - a total thickness of the subsurface strata; $\Delta-$ distance from the section line to an observation point; $v_{\max }$-velocity of displacement of the blocks near the rupture zone; $v(\Delta)$-velocity of displacement of blocks at a distance $\Delta$ from the rupture zone; $U_{k}(z, t)$ is a function of displacement in the direction parallel to the rupture; $\rho_{k}, G_{k}, H_{k}$-the density, modulus of shear, and a thickness of $k$ layer, respectively; $\left(H+H_{0}\right)$ is the depth of the rupture. 1-Direction of movement of the blocks after the rupture; 2-the direction of the rupture; 3-direction of the first inertial displacement of layers after the rupture (compression and extension in the medium).

After an instantaneous break (see Figure 2a), the soil particles of each block will have some initial velocity, the value of which will be determined by the length and depth of the rupture, the relative displacement $U_{0}$, and mechanical properties of the medium, that is, the earthquake seismic moment $M_{0}$ and, accordingly, the earthquake magnitude $M$. It is evident that soil particles will have the highest velocity near the rupture zone. By analogy with the abovementioned case of static deformation, one can assume that the initial motion velocity of soil particles at a certain distance from the rupture will be much lower than that near the rupture (Figure 2a). Proceeding from the existing theoretical studies and records of real strong earthquakes, we propose to take the following relation between the initial value of the velocity at a distance $\Delta$ from the rupture zone and the maximum velocity near it:
The process of breaking the medium usually occurs within a certain short period of time. In this paper we consider the case most unfavorable from the point of view of seismic action, where the break is considered to be instantaneous. (d)

$$
\mathrm{v}(\Delta)=\mathrm{v}_{\max }\left(1-\frac{\Delta^{2}}{R^{2}}\right),
$$

where values $R$, depending on the relative average displacement $\bar{U}_{0}$ are taken from the formula (1). According to Brun's research, accelerations exceeding $1 g$ and velocity $\mathrm{V}_{\max }$ of more than $100 \mathrm{~cm} / \mathrm{s}$ are possible in solid soil near an earthquake focus. He argues that the upper limit of the real initial velocity of soil particles in the majority of strong earthquakes is $150 \mathrm{~cm} / \mathrm{sec}$.

If we assume that this value $\mathrm{v}_{\max }=100 \mathrm{~cm} / \mathrm{sec}$ corresponds to the distance from the rupture zone of a strong earthquake with a magnitude of 8.5 and take into account that, according to [4], 
Table 1. The values of the velocities of soil particles vibration in $\mathrm{cm} / \mathrm{sec}$, depending on the magnitude $M$ and the distance from the rupture $\Delta$ in $\mathrm{km}$.

\begin{tabular}{|c|c|c|c|c|c|c|c|c|c|c|}
\hline \multirow{3}{*}{$\begin{array}{l}\text { Earthquake } \\
\text { magnitude M }\end{array}$} & \multirow{3}{*}{$\begin{array}{l}\text { Mean } \\
\text { slip } \bar{u}, \mathbf{m}\end{array}$} & \multirow{3}{*}{$\begin{array}{l}\text { Value of } R \\
\text { from }(1), \mathrm{km}\end{array}$} & \multirow{3}{*}{$\begin{array}{l}\text { Value velocities } \\
\text { for rupture } v_{\max } \text {, } \\
\mathrm{cm} / \mathrm{s}\end{array}$} & \multicolumn{7}{|c|}{$\begin{array}{l}\text { Value velocities } v(\Delta) \text { in } \mathrm{cm} / \mathrm{s} \text {, depending on the magnitude } M \text { and distance from } \\
\text { the rupture (3) }\end{array}$} \\
\hline & & & & \multicolumn{7}{|c|}{$\Delta$ in $\mathbf{k m}(\Delta<\mathbf{R})$} \\
\hline & & & & 5 & 10 & 15 & 20 & 25 & 30 & 35 \\
\hline 6.0 & 0.39 & 16.90 & 8.2 & 7.5 & 5.3 & 1.7 & & & & \\
\hline 6.25 & 0.54 & 17.70 & 10.5 & 9.7 & 7.1 & 3.0 & & & & \\
\hline 6.50 & 0.73 & 18.60 & 13.5 & 12.5 & 9.6 & 4.7 & & & & \\
\hline 6.75 & 1.00 & 20.00 & 17.4 & 16.3 & 13.1 & 7.6 & & & & \\
\hline 7.0 & 1.38 & 21.90 & 22.3 & 21.1 & 17.7 & 11.8 & 3.7 & & & \\
\hline 7.25 & 1.90 & 24.50 & 28.6 & 27.4 & 23.8 & 17.9 & 9.5 & & & \\
\hline 7.50 & 2.60 & 28.00 & 36.8 & 35.6 & 32.1 & 26.2 & 18.0 & 7.5 & & \\
\hline 7.75 & 3.57 & 32.80 & 47.2 & 46.1 & 42.8 & 37.3 & 29.7 & 19.8 & 7.7 & \\
\hline 8.0 & 4.90 & 39.50 & 60.6 & 59.6 & 56.7 & 51.9 & 45.1 & 36.3 & 25.6 & 13.0 \\
\hline 8.25 & 6.73 & 48.60 & 77.9 & 77.1 & 74.6 & 70.5 & 64.7 & 57.3 & 48.2 & 37.5 \\
\hline 8.50 & 9.23 & 61.10 & 100 & 99.3 & 97.3 & 94.0 & 89.3 & 83.3 & 75.9 & 67.2 \\
\hline 8.75 & 12.68 & 78.40 & 128.4 & 127.9 & 126.3 & 123.7 & 120.0 & 115.3 & 109.6 & 102.8 \\
\hline 9.0 & 17.38 & 102.00 & 164.9 & 164.5 & 163.3 & 161.3 & 158.6 & 155.0 & 150.6 & 145.5 \\
\hline
\end{tabular}

Table 1. Continue.

\begin{tabular}{|c|c|c|c|c|c|c|c|c|c|c|c|c|c|}
\hline \multirow{3}{*}{$\begin{array}{l}\text { Earthquake } \\
\text { magnitude M }\end{array}$} & \multicolumn{13}{|c|}{ Value velocities $v(\Delta)$ in $\mathrm{cm} / \mathrm{s}$, depending on the magnitude $M$ and distance from the rupture (3) } \\
\hline & \multicolumn{13}{|c|}{$\Delta$ in $\mathbf{k m}(\Delta<\mathbf{R})$} \\
\hline & 40 & 45 & 50 & 55 & 60 & 65 & 70 & 75 & 80 & 85 & 90 & 95 & 100 \\
\hline \multicolumn{14}{|l|}{6.0} \\
\hline \multicolumn{14}{|l|}{6.50} \\
\hline \multicolumn{14}{|l|}{6.75} \\
\hline \multicolumn{14}{|l|}{7.0} \\
\hline \multicolumn{14}{|l|}{7.25} \\
\hline \multicolumn{14}{|l|}{7.50} \\
\hline \multicolumn{14}{|l|}{7.75} \\
\hline 8.0 & & & & & & & & & & & & & \\
\hline 8.25 & 25.1 & 11.1 & & & & & & & & & & & \\
\hline 8.50 & 57.1 & 45.8 & 33.0 & 19.0 & 3.6 & & & & & & & & \\
\hline 8.75 & 95.0 & 86.1 & 76.2 & 65.2 & 53.2 & 40.1 & 26.0 & 10.9 & & & & & \\
\hline 9.0 & 139.5 & 132.8 & 125.3 & 117.0 & 107.8 & 97.9 & 87.2 & 75.7 & 63.5 & 50.4 & 36.5 & 21.9 & 6.4 \\
\hline
\end{tabular}

the maximum velocity of soil particles is proportional to $e^{M}$, where $M$ is an earthquake magnitude, the value $\mathrm{v}_{\max }$ at other magnitudes can be represented as follows:

$$
\mathrm{v}_{\max }=100 e^{M-8.5} \mathrm{~cm} / \mathrm{sec} .
$$

This equation enables us to evaluate the value $\mathrm{v}_{\max }$ on the surface near the rupture for strong earthquakes with a magnitude of $M>6.0$, in the case where the rupture is exposed on the surface. According to (2) and taking into account the above formula initial velocity of soil particles at a distance $\Delta$ from the rupture, we obtain:

$$
\mathrm{v}(\Delta)=100 e^{M-8,5}\left(1-\frac{\Delta^{2}}{R^{2}}\right) .
$$

Here is another empirical formula [6], linked to an earthquake magnitude of $M$ and relative motion $\bar{U}_{0}$ :

$$
\lg \bar{U}_{0}=0.55 M-3.71
$$

On the basis of formulas (1)-(4) values $\bar{U}_{0}(\mathrm{~m}), R(\mathrm{~km})$ and the corresponding values $v_{\max }(\mathrm{m} / \mathrm{sec})$ for different magnitudes $M$ were calculated (Table 1). The magnitudes not listed in Table 1 , namely the values of $U_{0}, R$, and $\mathrm{v}_{\max }$, were also calculated by the formulas (1)-(4).

In Table 2 are listed the values of the ground vibration velocities $(\mathrm{cm} / \mathrm{s})$, depending on the earthquake intensity on the MSK-64 scale. Comparison of Table 1 and Table 2 shows that the values of the ground particle velocities calculated by the formula (5) are comparable with their MSK-64 values for VII-X-point intensities in the epicentral zone, and in areas outside the zone with V-X-point intensity and $6.0 \leq \mathrm{M} \leq 8.0$ magnitudes earthquakes. 
Table 2. The values of the velocities $(\mathrm{cm} / \mathrm{s})$ of soil particles' oscillation depending on the earthquake intensity on the scale MSK-64.

\begin{tabular}{lllllll}
\hline Intensity & V & VI & VII & VIII & IX & X \\
\hline Velocity & $1 \div 2$ & $2.1 \div 4$ & $4.1 \div 8$ & $8.1 \div 16$ & $16.1 \div 32$ & $32.1 \div 64$ \\
\hline
\end{tabular}

Figure 3 shows the $\mathrm{v}(\Delta)$ dependences for some magnitudes $\mathrm{M}$ and earthquake intensity on the MSK-64 scale.

\subsection{Influence of Non-instantaneous Rupture}

In case of non-instantaneous destruction fracture, the initial value of soil particles' velocity, according to [1] can be represented as:

$$
\mathrm{v}=\mathrm{v}_{\mathrm{inst}} e^{-t / \Delta t}
$$

where: $v_{\text {inst }}$ is the above obtained value of the initial velocity at instantaneous destruction of the medium (3), $\Delta \mathrm{t}$ is the time of complete break, including the time of rupture, it can be written as:

\section{a}

$$
\Delta t=\frac{L / 2}{\mathrm{v}_{s}}
$$

where: $L$ is the length of the future rupture, $\mathrm{v}_{\mathrm{s}}$ is the propagation velocity of the transverse wave. The duration of destruction can last up to tens seconds. The ripping begins in the last stage of complete destruction, when a small part (within tens to hundreds meters) of the rupture line remains unbroken. Until this point, the dynamic effect of the earthquake - the generation of inertial forces in the ground has not yet manifested itself. The last stage of destruction (the beginning of the earthquake) -wrecking, according to experts $[1,15]$, occurs with supersonic speed and lasts a fraction of a second, within the range $t=0.1$ to 0.3 seconds (depending on the strength of rocks).

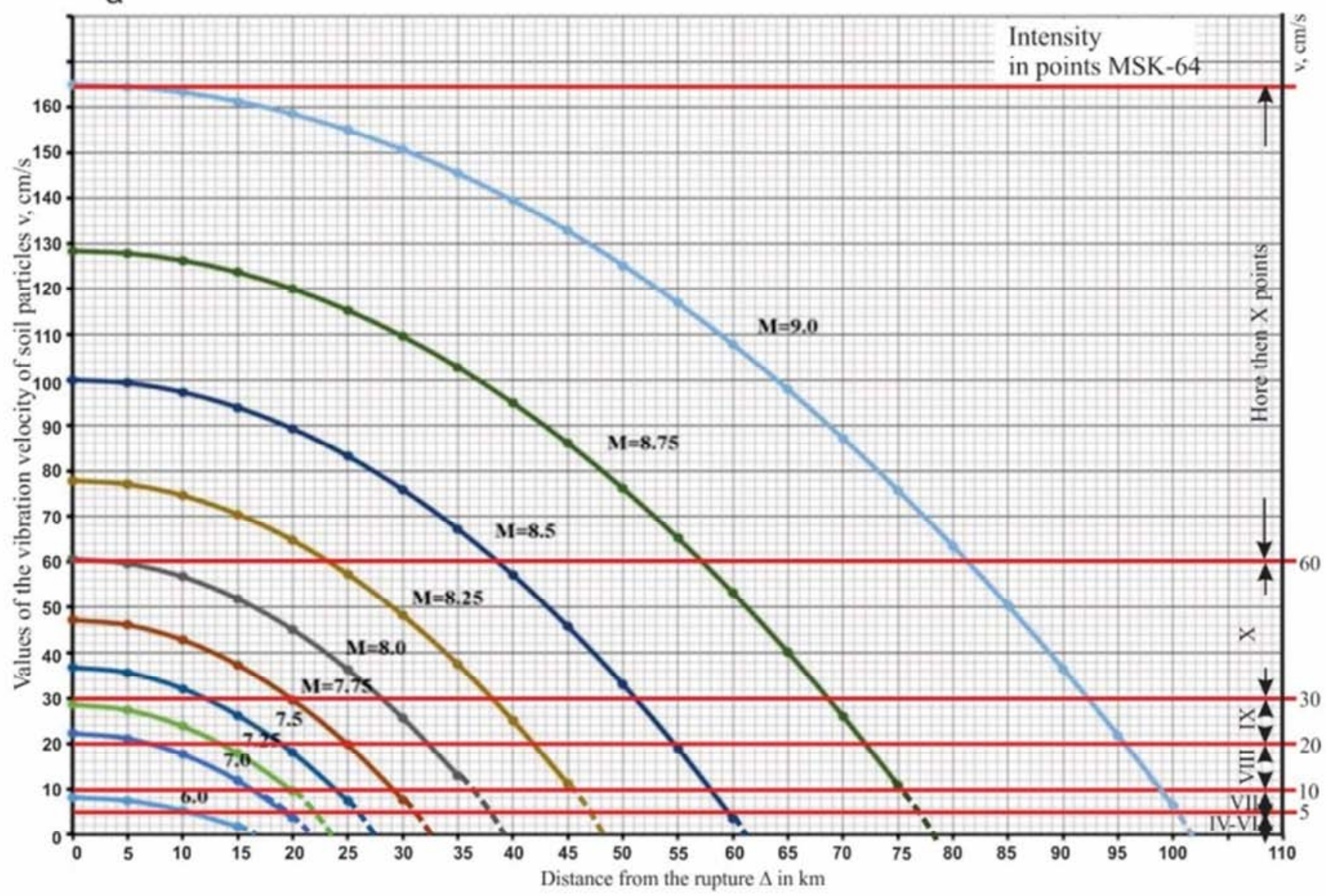




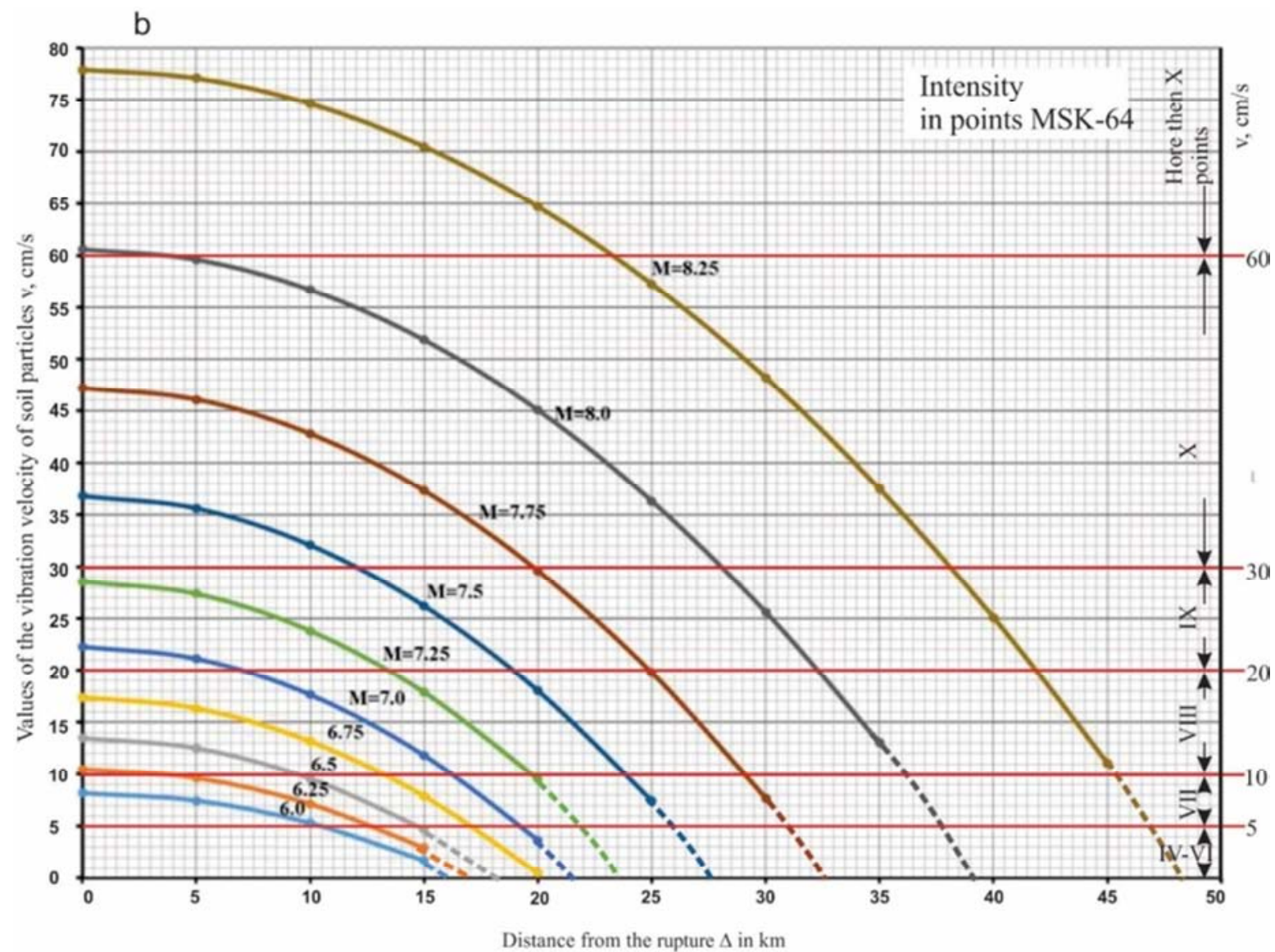

Figure 3. Dependences of the rates of velocity of soil particles from the epicentral distance $\triangle$ and intensity on the scale MSK-64, for different magnitudes. a.-for magnitudes $6.0 \leq M \leq 9.0$, b.- for magnitudes of $6.0 \leq M \leq 8.25$.

Therefore, by formula for $\mathbf{v}$, for example, we have:

$$
\begin{gathered}
\text { at } M=7.0, L=30 \mathrm{~km}, \mathrm{v}_{s}=4000 \mathrm{~m} / \mathrm{s}, \\
\Delta t=\frac{15 \cdot 1000}{4000}=3.75 \mathrm{~s}, \\
\text { at } M=8.0, L=80 \mathrm{~km}, \mathrm{v}_{s}=4000 \mathrm{~m} / \mathrm{s}, \\
\Delta t=\frac{40 \cdot 1000}{4000}=10 \mathrm{~s} .
\end{gathered}
$$

Assuming the ripping of the rupture, respectively, $t=0.1$ sec and $t=0.3 \mathrm{~s}$, according to the formula for $\mathbf{v}$, we obtain:

$$
\begin{aligned}
& \mathrm{v}=\mathrm{v}_{\text {inst }} e^{-\frac{0.1}{3.75}}=0.975 \mathrm{v}_{\text {inst }} \quad \text { in case } \mathrm{M}=7.0, \\
& \mathrm{v}=\mathrm{v}_{\text {inst }} e^{-\frac{0.3}{10}}=0.970 \mathrm{v}_{\text {inst }} \quad \text { in case } \mathrm{M}=8.0 .
\end{aligned}
$$

As we see, it is not the instantaneous discontinuity that leads to an insignificant decrease in the initial velocity of oscillation of soil particles and, consequently, to the same mitigation of the earthquake effect on the earth's surface.

\section{Mechanical Task Model}

The near surface rock layers differ significantly from deep ones in their kinematic conditions and physic mechanical properties. They are less dense, and their elasticity coefficients at extension $E$ and shear modulus $G$ are nearly twofold less than those of the underlying rocks. In addition, the surface layers are subject to essentially lower vertical compressive stress, their volume is insignificant compared to a total volume of ruptured blocks. Owing to these features, they are more subject to shear deformations in the horizontal and vertical directions compared to deep layers. Therefore, at instantaneous break of the crust, the layers of upper soil will be subject to much more intensive alternating dynamic displacements induced by the inertia of rocks than deep soil layers. During a real earthquake, seismographs and accelerographs record these alternating movements and accelerations of soil particles on the surface. (Static soil deformations occurring during the long-term preparation of an earthquake are not recorded by seismographs and accelerographs, as the base plates of their cases of a steady mass, held up by a spring, also move slowly together with the soil without a vibration of the inertial mass.)

If we chose a near surface prismatic area with a uniform width and a thickness of $H<<H_{0}$, where $H+H_{0}$ is the rupture depth (Figure 2a) in a block at a distance $\Delta$ from the rupture, then it presents a multilayer lithological column (stratum) in any part along the rupture length $L$ with different physic mechanical and geometrical characteristics: density $\rho_{k}$, shear modulus $G_{k}$, and thickness $H_{k}$ (Figure 2b). The depth $H$ means a distance from the surface (grade elevation of a construction) 
to dense rocks (as deep as $30 \mathrm{~m}$ ) at a propagation velocity of transverse seismic waves $\mathrm{v}_{S}$ of more than $1000 \mathrm{~m} / \mathrm{sec}$. There are numerous cases of simultaneous records of real earthquakes on the surface and at deep levels up to $150 \mathrm{~m}$, indicating a significant decrease of seismic effect at depth [5]. This is also confirmed by insignificant damage from strong earthquakes to underground structures [17]. It is also well known that the structure of the subsurface multilayer strata significantly affects the parameters of seismic vibrations.

Given the above, we assume that at the instantaneous break of the medium the influence of inertial movement of deep rock layers on that of surface layers can be neglected (Figure 2b). Thus, we consider a multilayer lithological column with a height $H$ (Figure $2 \mathrm{~b}-\mathrm{d}$ ), all layers of which at the moment $t=0$ have initial velocity $\mathrm{v}(\Delta)$, according to formula $(2 \mathrm{a})$ and the data given in Table 1, as the pattern for the calculation of variations displacements (seismogram) and accelerations (accelerogram) of soil particle movement on the surface over time.

\section{Mathematical Task Model and Its Solution}

The mathematical statement of the task is as follows. Firstly, let us find an expression of the piecewise constant function of the displacement of layers $U(z, t)$ (Figure 2c)

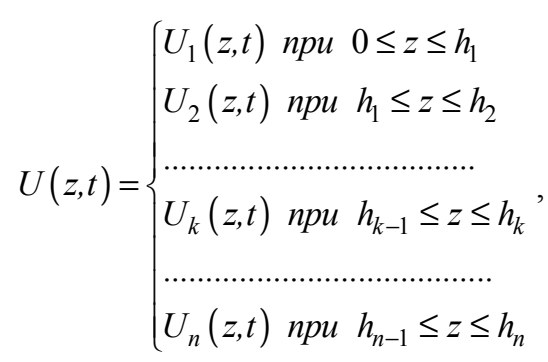

which satisfies the following wave equations of transverse shear vibrations of layers taking into account the viscosity of the medium $[10,18]$ :

$$
\begin{gathered}
G_{k} \frac{\partial^{2} U_{k}}{\partial z^{2}}-\rho_{k} \frac{\partial^{2} U_{k}}{\partial t^{2}}+\eta_{k} \frac{\partial^{3} U_{k}}{\partial z^{2} \partial t}=0, \\
k=1,2 \ldots n
\end{gathered}
$$

where $G_{k}$ is the shear modulus of rocks of $k$ layer; $\rho_{k}$, the density; $\eta_{k}$, the coefficient of viscosity; $H_{k}$, the thickness of layer $k$; $n$, the number of layers;

$$
h_{k}=\sum_{i=0}^{k} H_{i}, \quad h_{0}=0, \quad h_{1}=H_{1}, \quad h_{n}=H .
$$

Equation (6) must satisfy the following two boundary conditions on the surface $(z=0)$ and at a depth $H$ :

$$
\begin{array}{ll}
\text { at } z=0 & \frac{\partial U_{1}(0, t)}{\partial z}=0, \\
\text { at } z=H & U_{n}(H, t)=0,
\end{array}
$$

and $(2 n-2)$ conditions for continuity displacements and tangential stresses at the interfaces between layers:

$$
\begin{gathered}
U_{k}\left(h_{k}, t\right)=U_{k+1}\left(h_{k}, t\right) \\
G_{k} \frac{\partial U_{k}\left(h_{k}, t\right)}{\partial z}=G_{k+1} \frac{\partial U_{k+1}\left(h_{k}, t\right)}{\partial z} \\
k=1,2,3 \ldots n-1
\end{gathered}
$$

Equation (6) must also satisfy the following initial conditions:

$$
\begin{gathered}
\text { at } t=0 \quad U_{k}(z, 0)=0 \\
\text { at } t=0 \quad \frac{\partial U_{k}(z, 0)}{\partial t}=\mathrm{v}(\Delta) \\
k=1,2 \ldots n
\end{gathered}
$$

where $\mathrm{v}(\Delta)$ is the initial rate of motion of the base of a section.

The equations (6) will be solved using the method of separation of variables following the next formula:

$$
\begin{gathered}
U_{k}(z, t)=\sum_{i=1}^{\infty} U_{k i}(z) q_{i}(t) \\
k=1,2,3 \ldots n
\end{gathered}
$$

where $U_{k i}(z)$ is a function only of the coordinate $z$, and $q_{i}(t)$ is a function only of time $t$. Substituting (10) into (6) and taking into account that (10) must be satisfied for any $i$, we obtain

$$
G_{k} U_{k i}^{\prime \prime} q_{i}-\rho_{k} U_{k i} q_{i}^{\prime \prime}+\eta_{k} U_{k i}^{\prime \prime} q_{i}^{\prime}=0
$$

Dividing (11) by $U_{k i}\left(q_{i}+\frac{\eta_{k}}{G_{k}} q_{i}^{\prime}\right)$ we obtain the equation

$$
\frac{G_{k}}{\rho_{k}} \frac{U_{k i}^{\prime \prime}}{U_{k i}}=\frac{q_{i}^{\prime \prime}}{q_{i}+\frac{\eta_{k} q_{i}^{\prime}}{G_{k}}}=-p_{i}^{2}
$$

where $p_{i}^{2}$ is a positive number.

From (12) two equations follow:

$$
U_{k i}^{\prime \prime}+\lambda_{k i}^{2} U_{k i}=0 \sum_{k=1,2 \ldots n} \frac{p_{i}^{2} \rho_{k}}{G_{k}}=\lambda_{k i}^{2}
$$

$$
\begin{aligned}
q_{i}^{\prime \prime}+2 n_{i} q_{i}^{\prime}+p_{i}^{2} q_{i} & =0 \quad \frac{\eta_{k} p_{i}^{2}}{G_{k}}=2 n_{i} \\
i & =1,2,3 \ldots
\end{aligned}
$$

The solution of equation (14) will be as follows: 


$$
q_{i}(t)=e^{-n_{i} t}\left(c_{1 i} \sin p_{i}^{*} t+c_{2 i} \cos p_{i}^{*} t\right)
$$

where $c_{1 i}$ and $c_{2 i}$ are unknown constants and $p_{i}^{*}$ is circular frequencies of $i$-type free vibrations in an entire multilayer stratum with consideration of viscosity of rocks, which are determined by the formula:

$$
p_{i}^{*}=\sqrt{p_{i}^{2}-n_{i}^{2}}
$$

where $n_{i}$ is a critical damping coefficient of $i$ type free vibrations for the entire heterogeneous stratum. For the majority of rocks $n_{i}^{2} \ll p_{i}^{2}$, therefore, the influence of $n_{i}^{2}$ compared to $p_{i}^{2}$ on the angular frequencies in formula (16) and trigonometric functions of formula (15) can be neglected. Taking $\boldsymbol{p}_{\boldsymbol{i}}^{*} \approx \boldsymbol{p}_{\boldsymbol{i}}$, formula (16) can be as follows:

$$
q_{i}(t)=e^{-n_{i} t}\left(c_{1 i} \sin p_{i} t+c_{2 i} \cos p_{i} t\right)
$$

The solution of equation (13) can be written as

$$
U_{k i}(z)=A_{k i} \sin \lambda_{k i} z+B_{k i} \cos \lambda_{k i} z \quad k=1,2 \ldots n
$$

In order to determine $2 n$ of unknown coefficients $A_{k i}$ and $B_{k i}$ and circular frequencies $p_{i}$, let us use boundary conditions (7) and continuity conditions (8). As a result, we obtain the following system of $2 n$ homogeneous linear equations relative to $2 n$ of unknown coefficients of $A_{k i}$ and $B_{k i}$ :

$$
\begin{gathered}
A_{1 i}=0, \\
A_{n i} \sin \lambda_{n i} H+B_{n i} \cos \lambda_{n i} H=0, \\
A_{k i} \sin \lambda_{k i} h_{k}+B_{k i} \cos \lambda_{k i} h_{k}= \\
=A_{k+1 i} \sin \lambda_{k+1 i} h_{k}+B_{k+1 i} \cos \lambda_{k+1 i} h_{k}, \\
A_{k i} G_{k} \lambda_{k i} \cos \lambda_{k i} h_{k}-B_{k i} G_{k} \lambda_{k i} \sin \lambda_{k i} h_{k}= \\
=A_{k+1 i} G_{k+1} \lambda_{k+1 i} \cos \lambda_{k+1 i} h_{k}-B_{k+1 i} G_{k+1} \lambda_{k+1 i} \sin \lambda_{k+1 i} h_{k} \\
k=1,2 \ldots n-1 .
\end{gathered}
$$

Since the system of equations (19), relative to $2 n$ unknown coefficients $A_{k i}$ and $B_{k i}$ is homogeneous, there is a nontrivial solution only for the determinant of the $2 n$ degree being zero formed by unknown variable coefficients. In the case of a two-layer stratum it will be the determinant of the $4 n$ degree, for three and four-layer strata- $6 n$ and $8 n$ degrees, respectively. After expansion of the determinant, we obtain a complex transcendental equation containing only one unknown variable-the free vibration frequency $p_{i}^{2}$. For given $G_{k}, \rho_{k}$, and $H_{k}$ it serves as a basis for calculating the circular frequency $p_{i}$ $(i=1,2,3, \ldots)$ of all forms of free (predominant) vibrations of the multilayer thickness. It is almost impossible to get such an explicit equation for a large number of layers. Frequency values $p_{i}$ are often calculated using different computer programs directly from corresponding equations as the determinant of the system (19). For two-, three- and four-layer strata transcendental equations are given explicitly in $[9,10]$ :

$$
\text { at } n=2
$$

$$
\sqrt{\frac{\rho_{1} G_{1}}{\rho_{2} G_{2}}} \operatorname{tgp}_{i} \alpha_{1} \operatorname{tgp}_{i} \alpha_{2}-1=0 \text {, }
$$$$
\text { at } \mathbf{n}=\mathbf{3}
$$$$
\begin{gathered}
\sqrt{\frac{\rho_{1} G_{1}}{\rho_{2} G_{2}}} \operatorname{tg} p_{i} \alpha_{1} \operatorname{tg} p_{i} \alpha_{2}+\sqrt{\frac{\rho_{2} G_{2}}{\rho_{3} G_{3}}} \operatorname{tg} p_{i} \alpha_{2} \operatorname{tg} p_{i} \alpha_{3}+ \\
+\sqrt{\frac{\rho_{1} G_{1}}{\rho_{3} G_{3}}} \operatorname{tg}_{i} \alpha_{1} \operatorname{tg} p_{i} \alpha_{3}-1=0
\end{gathered}
$$

$$
\text { at } n=4
$$$$
\sqrt{\frac{\rho_{1} G_{1}}{\rho_{1} G_{2}}} \operatorname{tgp}_{i} \alpha_{1} \operatorname{tg} p_{i} \alpha_{2}+\sqrt{\frac{\rho_{1} G_{1}}{\rho_{3} G_{3}}} \operatorname{tgp}_{i} \alpha_{1} \operatorname{tgp}_{i} \alpha_{3}+
$$$$
+\sqrt{\frac{\rho_{1} G_{1}}{\rho_{4} G_{4}}} \operatorname{tgp}_{i} \alpha_{1} \operatorname{tg} p_{i} \alpha_{4}+\sqrt{\frac{\rho_{2} G_{2}}{\rho_{3} G_{3}}} \operatorname{tgp}_{i} \alpha_{2} \operatorname{tg} p_{i} \alpha_{3}+
$$$$
+\sqrt{\frac{\rho_{2} G_{2}}{\rho_{4} G_{4}}} \operatorname{tgp}_{i} \alpha_{2} \operatorname{tg} p_{i} \alpha_{4}+\sqrt{\frac{\rho_{3} G_{3}}{\rho_{4} G_{4}}} \operatorname{tgp}_{i} \alpha_{3} \operatorname{tg} p_{i} \alpha_{4}-
$$$$
-\sqrt{\frac{\rho_{1} G_{1} \rho_{3} G_{3}}{\rho_{2} G_{2} \rho_{4} G_{4}}} \operatorname{tg}_{i} \alpha_{1} \operatorname{tg} p_{i} \alpha_{2} \operatorname{tg} p_{i} \alpha_{3} \operatorname{tg} p_{i} \alpha_{4}-1=0
$$

at

$$
\alpha_{k}=H_{k} \sqrt{\frac{\rho_{k}}{G_{k}}}, \quad k=1,2,3 \ldots n
$$

Substituting the calculated frequency values $p_{i}=2 \pi / T_{0 \mathrm{i}}(i=1$, $2,3 \ldots$ ) into equations (20)-(22), we obtain systems of $n$ homogeneous linear equations relative to $A_{k i}$ and $B_{k i}$ with a major zero determinant. For nontrivial solution of the task we reject one of homogeneous equations and, then, given one of the unknown variables, for example, $B_{1 i}$, (in general, $B_{1 i}$ can be taken as equal to 1$)$ from the rest of the system $(2 n-1)$ of nonhomogeneous equations and determine all $(2 n-1)$ unknown relations of $A k i / B 1 i$ and $B k i / B 1 i$.

In order to determine the unknown coefficients $c_{1 i}$ and $c_{2 i}$ let us use the initial conditions (9). From the first condition (9), taking into account (10), (17), and (18), we have:

$$
\begin{gathered}
\sum_{i=1}^{\infty} U_{k i}(z) \cdot 1 \cdot\left(c_{1 i} \cdot 0+c_{2 i} \cdot 1\right)=0 \\
k=1,2 \ldots n
\end{gathered}
$$

Since the condition (23) must be valid for any point $z$ at the height of the lithological column, and coefficients of $A_{k i}$ and $B_{k i}$, as a part of $U_{k i}(z)$, can not be equal to zero, it follows from (23) that

$$
c_{2 i}=0, \quad i=1,2 \ldots
$$

From the second condition (9), we have 


$$
\begin{gathered}
\sum_{i=1}^{\infty} U_{k i}(z) \cdot 1 \cdot\left(\boldsymbol{c}_{1 i} \cdot p_{i} \cdot 1-p_{i} \cdot c_{2 i} \cdot 0\right)=\mathrm{v}(\Delta) \\
k=1,2 \ldots n
\end{gathered}
$$

Multiplying both sides of equation (24) by $\rho_{k}, U_{k j}(z)$ and integrating from zero to $\mathrm{H}$, replaced in advance with the sum of integrals from $h_{k-1}$ to $h_{k}$, in accordance with (5), we obtain:

$$
\sum_{k=1}^{n} \int_{h_{k-1}}^{h_{k}} \sum_{i=1}^{\infty} U_{k i}(z) \rho_{k} U_{k j}(z) c_{1 i} p_{i} d z=\mathrm{v}(\Delta) \sum_{k=1}^{n} \int_{h_{k-1}}^{h_{k}} \rho_{k} U_{k j}(z) d z
$$

Given the orthogonality of the function $U_{i}(z, t)$ and $U_{j}(z, t)$, according to [21]

$$
\sum_{k=1}^{n} \int_{h_{k-1}}^{h_{k}} \rho_{k} U_{k i}(z) U_{k j}(z) d z=0
$$

$$
i \neq j
$$

for all combinations of $i$ and $j$ vibrations, we obtain from (25)

$$
\boldsymbol{c}_{1 i}=\frac{\mathrm{v}(\Delta) \sum_{k=1}^{n} \int_{h_{k-1}}^{h_{k}} \rho_{k} U_{k i}(z) d z}{p_{i} \sum_{k=1}^{n} \int_{h_{k-1}}^{h_{k}} \rho_{k} U_{k i}^{2}(z) d z} .
$$

Thus, based on (10), (17), (18), and (27), the general solution of the task, taking into account all modes of vibration in the soil stratum, is as follows:

$$
U_{k}(z, t)=\mathrm{v}(\Delta) \sum_{i=1}^{\infty} \frac{U_{k i}(z)}{p_{i}} \delta_{i} e^{-n_{i} t} \sin p_{i} t
$$

Given the relative smallness of the damping coefficient $n_{i}$, for acceleration $U_{k}^{\prime \prime}(z, t)$ we obtain from (28)

$$
\begin{gathered}
U_{k}^{\prime \prime}(z, t)=\mathrm{v}(\Delta) \sum_{i=1}^{n} U_{k i}(z) p_{i} \delta_{i} e^{-n_{i} t} \sin p_{i} t \\
k=1,2 \ldots n \\
\text { where: } \delta_{i}=\frac{\sum_{k=1}^{n} \int_{h_{k-1}}^{h_{k}} \rho_{k} U_{k i}(z) d z}{\sum_{k=1}^{n} \int_{h_{k-1}}^{h_{k}} \rho_{k} U_{k i}^{2}(z) d z} .
\end{gathered}
$$

For motion of soil particles (seismograms) and acceleration (accelerograms) on the surface we have

$$
\begin{aligned}
& U_{1}(0, t)=\mathrm{v}(\Delta) \sum_{i=1}^{\infty} \frac{U_{1}(0)}{p_{i}} \delta_{i} e^{-n_{i} t} \sin p_{i} t \\
& U_{1}^{\prime \prime}(0, t)=\mathrm{v}(\Delta) \sum_{i=1}^{\infty} U_{1}(0) p_{i} \delta_{i} e^{-n_{i} t} \sin p_{i} t
\end{aligned}
$$

For the most simple (one layer) stratum with parameters of $G, \rho, H$ (Figure $2 \mathrm{~d}$ ) the task is essentially simpler:

$$
U(z, t)=U_{1}(z, t) \quad 0 \leq z \leq H
$$

For one-layer stratum we have the next equation satisfying the boundary and initial conditions (7) and (9)

$$
\begin{gathered}
U_{i}(z)=B_{i} \cos \frac{2 i-1}{2} \frac{\pi}{H} z, \quad A_{i}=0, \quad c_{2 i}=0, \quad c_{1 i} B_{i}=\frac{\mathrm{v}(\Delta) \delta_{i}}{p_{i}}, \\
p_{i}=\frac{2 \pi}{T_{0 i}}=\frac{2 i-1}{2 H} \pi \sqrt{\frac{G}{\rho}}=\frac{2 i-1}{2 H} \pi \mathrm{v}_{s}, i=1,2, \ldots, \\
\delta_{i}=\frac{\int_{0}^{H} \cos \frac{2 i-1}{2} \frac{\pi}{H} z d z}{\int_{0}^{H} \cos ^{2} \frac{2 i-1}{2} \frac{\pi}{H} z d z}=\frac{4(-1)^{i+1}}{(2 i-1) \pi},
\end{gathered}
$$

and the general solution is as follows:

$$
\begin{aligned}
& U(z, t)=\mathrm{v}(\Delta) \sum_{i=1}^{\infty} \frac{T_{o i}}{2 \pi} \delta_{i} e^{-n_{i} t} \cos \frac{2 i-1}{2} \frac{\pi z}{H} \sin \frac{2 i-1}{2} \frac{\pi \mathrm{v}_{s}}{H} t \\
& U^{\prime \prime}(z, t)=\mathrm{v}(\Delta) \sum_{i=1}^{\infty} \frac{2 \pi}{T_{o i}} \delta_{i} e^{-n_{i} t} \cos \frac{2 i-1}{2} \frac{\pi z}{H} \sin \frac{2 i-1}{2} \frac{\pi \mathrm{v}_{s}}{H} t
\end{aligned},
$$

where $T_{o i}=\frac{2 \pi}{p_{i}}=\frac{4 H}{(2 i-1) \mathrm{v}_{s}}$ is the period of $\mathrm{i}$ type free vibrations in a layer; $n i$ is the critical damping coefficient of $i$ type free vibrations in a layer; and $\mathrm{v}_{s}=\sqrt{G / \rho}$ is the propagation velocity of transverse waves in a layer.

As can be seen from formulas (28) and (33), the main difference between homogeneous and heterogeneous strata (construction sites) is coefficients $\delta_{i}$ and relations of $T_{o 1} / T_{o 1}, T_{o 1} / T_{o 2}, \ldots, T_{o 1} / T_{o n}$. For a homogenous layer these relations (see (28)) are odd numbers $1,3,5,7, \ldots,(2 n-1)$, and the sign of coefficients $\delta_{i}$ for even $i$ will be "+", whereas for odd $i$ it will be "-". Therefore, for the summation of series (33), signs $\delta_{i}$ and maximums of trigonometric functions $\sin \frac{2 i-1}{2} \frac{\pi \mathrm{v}_{s} t}{H}$ for any $i$ will be the same. This leads to their algebraic summation and a significant increase in the total maximum value of the series. The probability of such coincidence for a heterogeneous column is very small, which results in the increase of the total of the series. Formula (33) can be interpreted in terms of waves. It is known that a real seismogram is the result of the summation of transverse seismic waves repeatedly refracted and reflected from lower 
layers and the surface. This conclusion also follows from formulas (28) and (33). In fact, we use the well-known statement [3] that any standing wave can be replaced by a pair of traveling waves, as follows from the equality

$$
2 \cos \frac{k \pi}{H} z \sin \frac{k \pi}{H} c t=\sin \frac{k \pi}{H}(z+c t)+\sin \frac{k \pi}{H}(c t-z),
$$

and, on the contrary, any traveling wave can be represented as a pair of standing waves with phases shifted by $\pi / 2$, since

$$
\sin \frac{k \pi}{H}(z+c t)=\sin \frac{k \pi z}{H} \cos \frac{k \pi c t}{H}+\cos \frac{k \pi z}{H} \sin \frac{k \pi c t}{H},
$$

where $c$ is the velocity of wave propagation. Then, the solution of (33) can be represented in the form of a wave:

$$
\begin{gathered}
U(z, t)=\mathrm{v}(\Delta) \sum_{i=1}^{\infty} \frac{T_{o i} \delta_{i}}{4 \pi} e^{-n_{i} t} \times \\
\times\left[\sin \frac{2 i-1}{2} \frac{\pi}{H}\left(z+\mathrm{v}_{s} t\right)+\sin \frac{2 i-1}{2} \frac{\pi}{H}\left(\mathrm{v}_{s} t-z\right)\right] \\
U^{\prime \prime}(z, t)=\mathrm{v}(\Delta) \sum_{i=1}^{\infty} \frac{\pi}{T_{o i}} \delta_{i} e^{-n_{i} t} \times \\
\times\left[\sin \frac{2 i-1}{2} \frac{\pi}{H}\left(z+\mathrm{v}_{s} t\right)+\sin \frac{2 i-1}{2} \frac{\pi}{H}\left(\mathrm{v}_{s} t-z\right)\right]
\end{gathered}
$$

where $\mathrm{v}_{s}$ is the velocity of transverse waves in a homogeneous layer.

As follows from equation (36), the seismogram and accelerogram of the soil represent the sum of a set of incident and reflected from the surface waves with varying amplitudes and frequencies.

According to modern concepts of the soil dynamics, there are the following relations between the critical damping coefficients $n(\%)$, the damping decrement $\theta$, the absorption coefficient $\psi$, and free oscillation period $T_{0}$ :

$$
\psi=2 \theta, n=\frac{\theta}{T_{o}}
$$

The water saturation of the soil leads to a nearly twofold increase in the decrement of damping $\theta$ compared with that in the dry soil. The $\theta$ value of dry sands at mean strain reaches 0.2 (Faccioli and Reséndiz, 1976). According to experimental studies by Stavnitser the values for different types of the soil with a density $\left(1.63 \leq \rho\left(\operatorname{ton} / \mathrm{m}^{3}\right) \leq 2.12\right)$ and a level of humidity from 5 to $30 \%$, the $\theta$ value changes from 0.17 to 0.64 [20].

Thus, equations describing soil displacement on the surface (at $z=0$ ) (seismogram) and its acceleration (accelerogram) at a distance $\Delta$ from the rupture zone at a magnitude $M$ of a predicted earthquake taking into consideration (3) and (31) for a one layer base will be:

$$
\begin{aligned}
& U(0, t)=100 e^{M-8.5}\left(1-\frac{\Delta^{2}}{R^{2}}\right) \sum_{i=1}^{\infty} \frac{T_{o i}}{2 \pi} \delta_{i} e^{-\frac{\theta_{i}}{T_{o i}} t} \sin \frac{2 \pi}{T_{o i}} t \\
& U^{\prime \prime}(0, t)=100 e^{M-8.5}\left(1-\frac{\Delta^{2}}{R^{2}}\right) \sum_{i=1}^{\infty} \frac{2 \pi}{T_{o i}} \delta_{i} e^{-\frac{\theta_{i}}{T_{o i}} t} \sin \frac{2 \pi}{T_{o i}} t
\end{aligned}
$$

where the value $R$, depending on a magnitude $M$, is taken from Table 1.

Equations (31) allow us to take any other relations and conditions for the given area, instead of value $\mathrm{v}(\Delta)(3)$, since it is included in (31) as a constant time independent $(t)$ factor. Due to this, it can not influence the summation of the modes of vibration.

According to (38), the acceleration value in homogeneous solid soil at given $M$ and $\Delta$ is higher than that of a homogeneous loose soil layer of the same thickness. This phenomenon has been repeatedly confirmed by instrumental records during weak and moderate earthquakes. Since the amplitudes of all harmonics (38) are greatly reduced over time, when searching for the maximums of expressions of $U(t)$ and $U^{\prime \prime}(t)$ one can only take into consideration the first three members of series (the first three modes of free transverse vibrations in a layer of $H$ thickness). Thus, acceleration of the soil displacement on the surface (and in depth of a layer) will be a superposition of dumping harmonic oscillations with periods equal to those of free oscillations in a layer $T_{0 i}$.

Another feature of these formulas (36) is that acceleration of soil displacement depends essentially on its seismic type, which is determined by values of predominant periods $T_{0 i}$ of a construction site in formulas (38). In order to determine the seismic type of the soil, it is recommended [10] to use, in addition to a mean value $\overline{\mathrm{v}}_{s}$, the period value $T_{01}$ as an integral characteristic of heterogeneous soils, as was discussed above, since it is determined by physic mechanical features and the thickness values of all layers. In addition, it is known that the relations of periods of induced (soil) and free vibrations (construction) play a significant role for all dynamic effects. In accordance with accepted standards of earthquake engineering in the Republic of Armenia [19], soil type is established according to values of $\overline{\mathrm{v}}_{s}$ and $T_{01}$ [7]) (Table 3). Thus, in the case of a large number of layers, approximate values $\boldsymbol{T}_{\boldsymbol{o l}}$ and mean value $\overline{\mathbf{v}}_{\boldsymbol{s}}$ are recommended to be determined by formulas

$$
T_{o 1}=\frac{4 H}{\overline{\mathrm{v}}_{s}}, \quad \overline{\mathrm{v}}_{s}=\frac{\sum_{k=1}^{n} H_{k}}{\sum_{k=1}^{n} \frac{H_{k}}{\mathrm{v}_{s k}}}, H=\sum_{k=1}^{n} H_{k}, \quad \mathrm{v}_{s k}=\sqrt{\frac{G_{k}}{\rho_{k}}} .
$$

The main feature of formulas (38) is that based on a predicted earthquake magnitude $M$ given and a known distance from an observation point before rupture (active fault) $\Delta$ one can evaluate not only maximum displacement and acceleration values of the soil, taking into account the soil conditions and variations of these parameters over time and, 
which is quite important, the vibration periods. In this case, according to (38), short-period vibrations will dominate in solid soil, and long period vibrations, in loose soil.

The main feature of any complex vibration process is its frequency spectrum. In our opinion, vibration periods (on accelerograms) play a major role in the behavior of the aboveground parts of a building construction during an earthquake. Seismic impact belongs among the dynamic effects where resonance phenomena (the coincidence or similarity of vibration periods in the soil and free vibrations in the aboveground parts of constructions) induce the greatest effect. There are numerous studies considering values of predominant periods of strong earthquake induced soil vibrations $[5,10,23]$, according to which the spectra of earthquake response (the maximum acceleration values on the spectrum) based on records of real soil accelerograms obtained in areas with different geological conditions, which are regarded as a source of the most general information about these values. At the current time, there are a large number of such spectra recorded. A comparative analysis shows that predominant periods for solid soil during an earthquake are mainly in a narrow range, from 0.15 to $0.4 \mathrm{sec}$, whereas those for the loose soil are in a wide range, from 0.5 to $2.0 \mathrm{sec}$, sometimes reaching $3.0 \mathrm{sec}$ or more.

The values of predominant periods $[7,10]$ determined by formulas (20)-(22) for different geological sections (with the values of physical and mechanical properties of layers $\rho_{k}, G_{k}$, and $H_{k}$, obtained during experimental drilling) and their comparison with predominant periods of peak acceleration values on response spectra for strong earthquakes recorded in the same sections [2] have good correlation.

Table 3. Soil types, according to their seismic properties in dependence on $\overline{\mathrm{v}}_{S}$ and $T_{01}$, according to $[10,19]$.

\begin{tabular}{lll}
\hline Type & $\overline{\mathrm{v}}_{\boldsymbol{s}}, \mathbf{m} / \mathbf{s e c}$ & $\boldsymbol{T}_{\mathbf{0 1}}, \mathbf{s e c}$ \\
\hline I & $>800$ & $\leq 0.3$ \\
II & $500 \leq \overline{\mathrm{v}}_{\boldsymbol{s}} \leq 800$ & $0.3<T_{01} \leq 0.6$ \\
III & $150<\overline{\mathrm{v}}_{\boldsymbol{s}}<500$ & $0.6<T_{01} \leq 0.8$ \\
IV & $<150$ & $>0.8$ \\
\hline
\end{tabular}

$\overline{\mathrm{v}}_{\boldsymbol{S}}$-is the mean velocity of transversal wave propagation within the entire heterogeneous stratum $\mathrm{H}$, from the grade elevation of a construction to dense rocks at $\overline{\mathrm{V}}_{s} \geq 800 \mathrm{~m} / \mathrm{sec}$ according to formula (39); $\mathrm{T}_{01}$ is the predominant period for the entire stratum $\mathrm{H}$ from the grade elevation to dense rocks with $\overline{\mathrm{v}}_{s} \geq 800 \mathrm{~m} / \mathrm{sec}$.

The maximum (the largest of the maximum values) acceleration value in the response spectrum can be recorded during the first predominant period $T_{01}$, on the one hand, and during higher periods of $T_{02}$ and $T_{03}$, on the other hand. As shown in [17], the real values of prevailing periods for earthquakes with $M>6$ are close to those determined by a harmonic analysis of soil micro vibrations at the observation site. It should be noted that there are other works [16], showing that the type of displacement in the distance from the rupture zone, magnitude, and distance from the rupture distance greatly affect the formation of values of predominant periods.

It should be also noted that the values of predominant vibration periods in the soil and free vibrations of buildings and structures of mass construction are in the same range of $0.1-1.0 \mathrm{sec}$. This fact essentially increases the probability of the occurrence of resonance phenomena. In addition, this probability for strong earthquakes increases due to their longer duration. In our opinion, resonance vibrations are the reason for most cases of collapses and serious damages to buildings and constructions during strong earthquakes. This is confirmed by the serious damage to individual constructions recorded during earthquakes at large distances from their epicenters, even at low levels of soil acceleration amplitudes $(<0.1 \mathrm{~g})$. The destruction recorded during earthquakes in 1985 in Mexico City and the Spitak earthquake in 1988 in towns of Leninakan and Kirovakan, are regarded as classical examples $[2,10]$.

The above means that the design of new buildings and structures should be preceded by reliable prediction of not only the maximum amplitude of soil acceleration, but also the value of predominant periods of vibrations during an earthquake. This prediction will allow the choice of the most favorable ground sites for each construction with a certain frequency spectrum of own vibrations. According to [10, 19], this condition is expressed by the following inequality:

$$
T_{1}>1.5 T_{01} \text { or } 1.5 T_{1}<T_{01},
$$

where $T_{1}$ is the period of the first mode of free vibrations of the aboveground part of a building construction, and $T_{01}$ is the dominant vibration period in a soil layer by formula (20)-(22). Since buildings and structures and their multilayered basements have signs of shear deformation, the relations between the values of periods of higher modes of vibration in both types of objects are the same. This suggests that having avoided resonance in construction for the first mode of vibration, one can avoid resonance vibrations for higher modes of vibration.

\section{Application of the Proposed Method of Prediction of the Surface Ground Vibration Parameters}

\subsection{Synthetic Seismograms and Accelerograms of Various Grounds}

As illustrating examples, seismograms and accelerograms of the base for soils seismic properties of I-IV categories with the predominant periods from $0.1 \mathrm{sec}$ to $2.0 \mathrm{sec}$, according to the code of quakeproof construction of the Republic of Armenia [19] have been calculated (Table 3.). The summary data of the maximum values $U_{\max }$ and $U_{\max }^{\prime \prime}$ obtained by formulas (38), taking into account the three forms of oscillations and only in the first form at $M=7.0, \Delta=15 \mathrm{~km},(R$ $=21.9 \mathrm{~km}$ ), given in Table 4 . The corresponding artificial seismograms and accelerograms are presented in Figures. 4 and 5 . 
For a uniform surface layer, as seen from (32), the values of oscillation periods $T_{0 \mathrm{i}}$ differ in 3 and 5 times, therefore at the that point of occurrence of the maximum amplitudes of the individual terms of the series (38) coincide in time and in the direction (the signs $\delta_{i}$ ). This leads to a significant increase in the total value of the series, especially for accelerations $U^{\prime \prime}(t)$. This is shown graphically in Figure 6 for acceleration $U^{\prime \prime}(t)$ calculated by the formula (38) by the first, second and third wave forms individually and in their combined action (superposition) for the case of $T_{01}=0.25 \mathrm{~s}$ at the same input parameters: $M=7.0, \Delta=15 \mathrm{~km}, \Theta_{1}=\Theta_{2}=\Theta_{3}=0.3$.

As can be seen the data listed in Table 4 and shown in Figures 4 . and 5 for hard soils $\left(T_{01}<0.45 \mathrm{~s}\right)$ and loose soils $\left(T_{01}>0.45 \mathrm{~s}\right)$, taking into consideration the higher forms of oscillation of the base leads to an increase in the maximum values of the surface accelerations by 2.53 times on the average, calculated only for the first form of oscillation, and just by 1.1 times for maximum values of ground displacements.

At the same time, the duration of intensive oscillations on loose soils is up to 2 times greater than on hard soils. One of the reasons for this phenomenon is that the displacements directly depend on the periods $T_{0 \mathrm{i}}$, and accelerations are inversely proportional to $T_{0 \mathrm{i}}$. In addition, for hard soils, $\left(T_{01}=\right.$ $0.25 \mathrm{~s}$ ), the periods of the second and third forms of oscillation, according to the formula (32) are: $T_{02}=0.083 \mathrm{~s}, T_{03}=0.05 \mathrm{~s}$. It is readily seen that they are of higher-frequency in comparison with their values for loose soils. Several investigators consider the occurrence of such high-frequency oscillations on the ground surface highly unlikely event and does not pose a danger to most of the above-ground structures. Therefore, apparently, their presence in formulas (38) for rocky soils with periods $T_{02}$ and $T_{03}$ is less than $0.1 \mathrm{~s}$, can be considered unrealistic, i.e. for the calculated value of the acceleration of solid soils with periods $\mathrm{T}_{01} \leq 0.25 \mathrm{~s}$, can be taken only taking into account the first form of oscillations. For the examined example it will be $0.36 \mathrm{~g}$. Let us note one more circumstance in favor of such a conclusion. The values of the internal friction coefficients $n_{2}$ and $n_{3}$ for the second and third waveforms (due to the lack of experimental studies relating to the processes of internal friction in the higher forms of transverse rock vibrations) were adopted both for the first form, which can also lead to great errors.

As far as this phenomenon is concerned there are different views in the literature stating that even if such high-frequency oscillations on the ground surface occur, they quickly decay and may have some effect on the formation of the total accelerogram of the soil only in a narrow focal zone of the earthquake.

According to the nature of the oscillations, the artificial seismograms and accelerograms shown in Figure 4.5 are close to the seismograms and accelerograms obtained in real earthquakes in Port Gueneme on March 18, 1957 and in Park field on June 27, 1966, presented in works [8, 17].

Table 4. Maximum values of displacements $U_{\max }$ and accelerations $U^{\prime \prime}{ }_{\max }$ of the soil at magnitude $M=7.0$ and at the distance $\Delta=15 \mathrm{~km}$, depending on the predominant base period $T_{01}$.

\begin{tabular}{|c|c|c|c|c|c|}
\hline \multirow[b]{2}{*}{$\begin{array}{l}\text { The predominant period of the } \\
\text { base oscillation } T_{01} \text {, sec }\end{array}$} & \multirow{2}{*}{$\begin{array}{l}\text { Damping decrement of } \\
\text { the ground, } \Theta\end{array}$} & \multirow{2}{*}{$\begin{array}{l}\text { The base category by seismic } \\
\text { properties according to [19] }\end{array}$} & \multicolumn{3}{|c|}{ Given the three forms of oscillation by (38) } \\
\hline & & & $\begin{array}{l}\text { Maximization } \\
\text { time t, s }\end{array}$ & $\begin{array}{l}\text { Maximum } \\
\text { displacement } U_{\max }, \mathrm{cm}\end{array}$ & $\begin{array}{l}\text { Maximum acceleration } U \\
{ }_{\text {max }} \text {, in fractions of } g\end{array}$ \\
\hline 0.1 & 0.3 & I & 0.02 & 0.23 & 1.34 \\
\hline 0.15 & 0.3 & I & 0.04 & 0.37 & 1.45 \\
\hline 0.25 & 0.3 & I & 0.06 & 0.63 & 0.92 \\
\hline 0.3 & 0.3 & II & 0.08 & 0.74 & 0.72 \\
\hline 0.35 & 0.3 & II & 0.08 & 0.87 & 0.61 \\
\hline 0.45 & 0.2 & II & 0.12 & 1.14 & 0.52 \\
\hline 0.6 & 0.2 & III & 0.15 & 1.6 & 0.42 \\
\hline 0.7 & 0.2 & III & 0.18 & 1.8 & 0.35 \\
\hline 0.8 & 0.2 & III & 0.21 & 2.04 & 0.30 \\
\hline 1.0 & 0.2 & IV & 0.24 & 2.56 & 0.25 \\
\hline 1.2 & 0.2 & IV & 0.3 & 3.1 & 0.21 \\
\hline 1.4 & 0.2 & IV & 0.36 & 3.61 & 0.18 \\
\hline 2.0 & 0.2 & IV & 0.48 & 5.16 & 0.12 \\
\hline
\end{tabular}

Table 4. Continue.

\begin{tabular}{|c|c|c|c|c|}
\hline \multirow{2}{*}{$\begin{array}{l}\text { The predominant period of the } \\
\text { base oscillation } T_{01} \text {, sec }\end{array}$} & \multicolumn{2}{|c|}{$\begin{array}{l}\text { Taking into account only the first form of } \\
\text { oscillation, by (38) }\end{array}$} & \multicolumn{2}{|c|}{$\begin{array}{l}\text { Relations between displacements and accelerations, taking into account } \\
\text { the three forms of oscillations to their values, only for the first form }\end{array}$} \\
\hline & $U_{\max }, \mathbf{c m}$ & $U^{\prime \prime}{ }_{\max }$, in fractions of $g$ & For displacements & For accelerations \\
\hline 0.1 & 0.22 & 0.90 & 1.0 & 1.5 \\
\hline 0.15 & 0.33 & 0.60 & 1.1 & 2.4 \\
\hline 0.25 & 0.56 & 0.36 & 1.1 & 2.6 \\
\hline 0.3 & 0.67 & 0.30 & 1.1 & 2.4 \\
\hline 0.35 & 0.78 & 0.26 & 1.1 & 2.4 \\
\hline 0.45 & 1.03 & 0.20 & 1.1 & 2.5 \\
\hline 0.6 & 1.37 & 0.15 & 1.2 & 2.7 \\
\hline 0.7 & 1.60 & 0.13 & 1.1 & 2.7 \\
\hline
\end{tabular}




\begin{tabular}{|c|c|c|c|c|}
\hline \multirow{2}{*}{$\begin{array}{l}\text { The predominant period of the } \\
\text { base oscillation } T_{01} \text {, sec }\end{array}$} & \multicolumn{2}{|c|}{$\begin{array}{l}\text { Taking into account only the first form of } \\
\text { oscillation, by (38) }\end{array}$} & \multicolumn{2}{|c|}{$\begin{array}{l}\text { Relations between displacements and accelerations, taking into account } \\
\text { the three forms of oscillations to their values, only for the first form }\end{array}$} \\
\hline & $U_{\max }, \mathbf{c m}$ & $U^{\prime \prime}{ }_{\max }$, in fractions of $g$ & For displacements & For accelerations \\
\hline 0.8 & 1.83 & 0.12 & 1.1 & 2.6 \\
\hline 1.0 & 2.28 & 0.09 & 1.1 & 2.7 \\
\hline 1.2 & 2.74 & 0.08 & 1.1 & 2.7 \\
\hline 1.4 & 3.20 & 0.07 & 1.1 & 2.7 \\
\hline 1.7 & 3.88 & 0.05 & 1.1 & 2.7 \\
\hline 2.0 & 4.57 & 0.05 & 1.1 & 2.7 \\
\hline
\end{tabular}
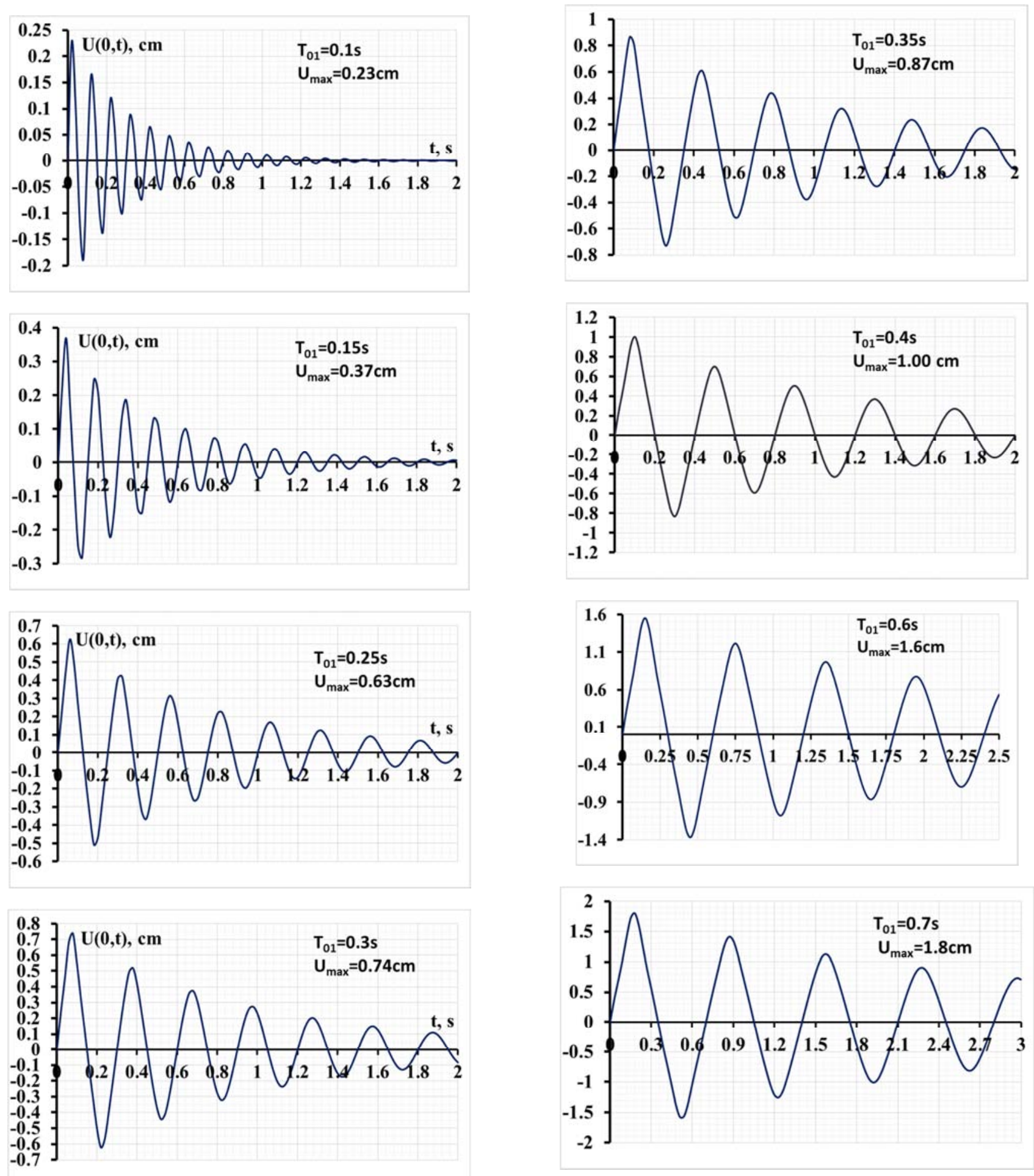

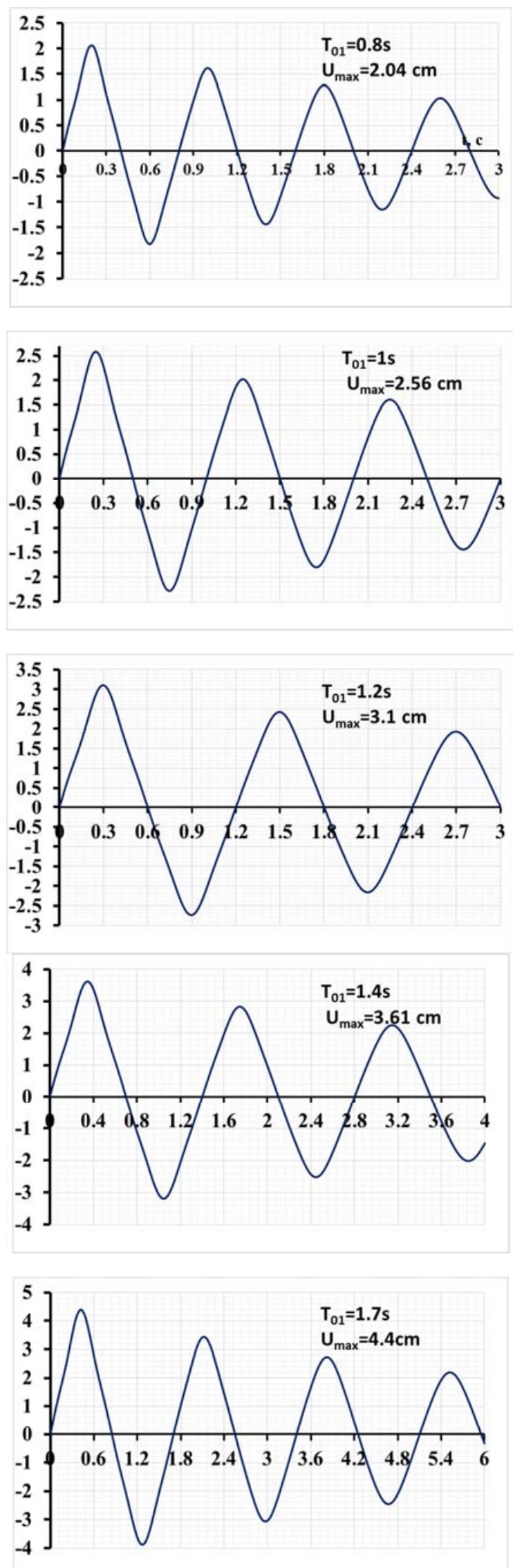

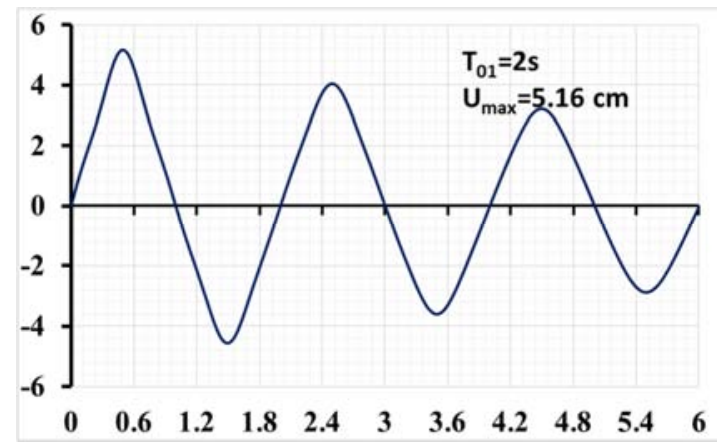

Figuire 4. Synthetic seismograms of various bases $\left(T_{01}\right)$, at $M=7, \Delta=15 \mathrm{~km}$.
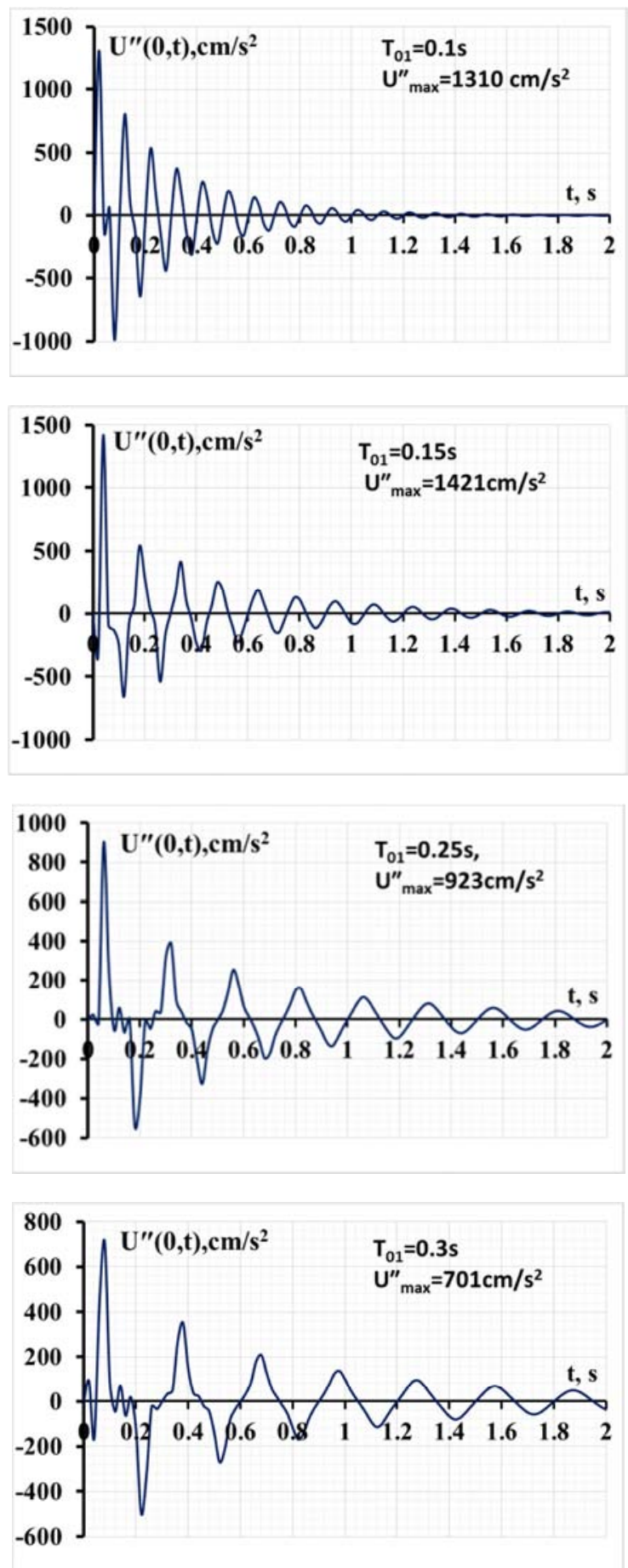

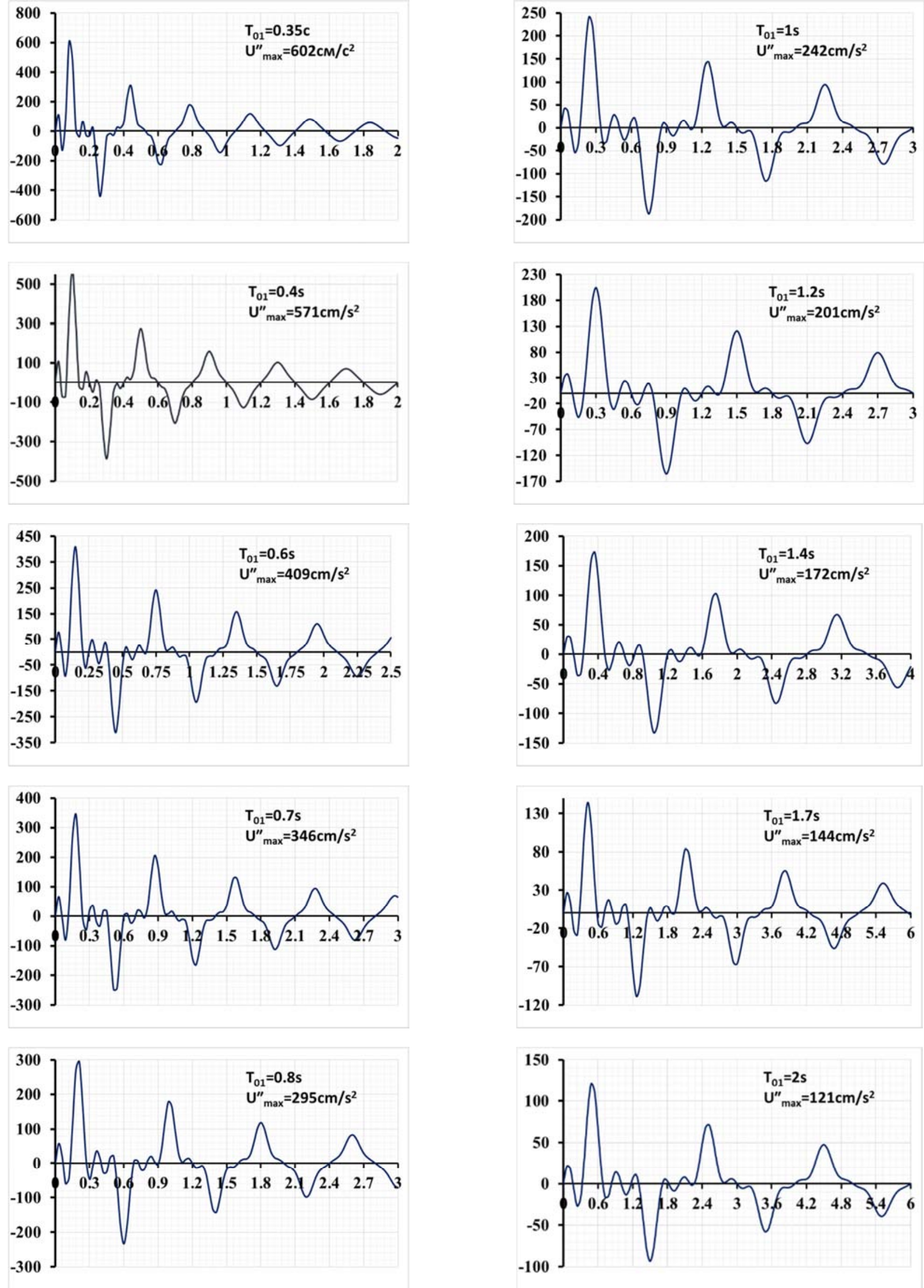

Figure 5. Synthetic accelerograms of various bases (T01) at $M=7.0, \Delta=15$ $\mathrm{km}$. 


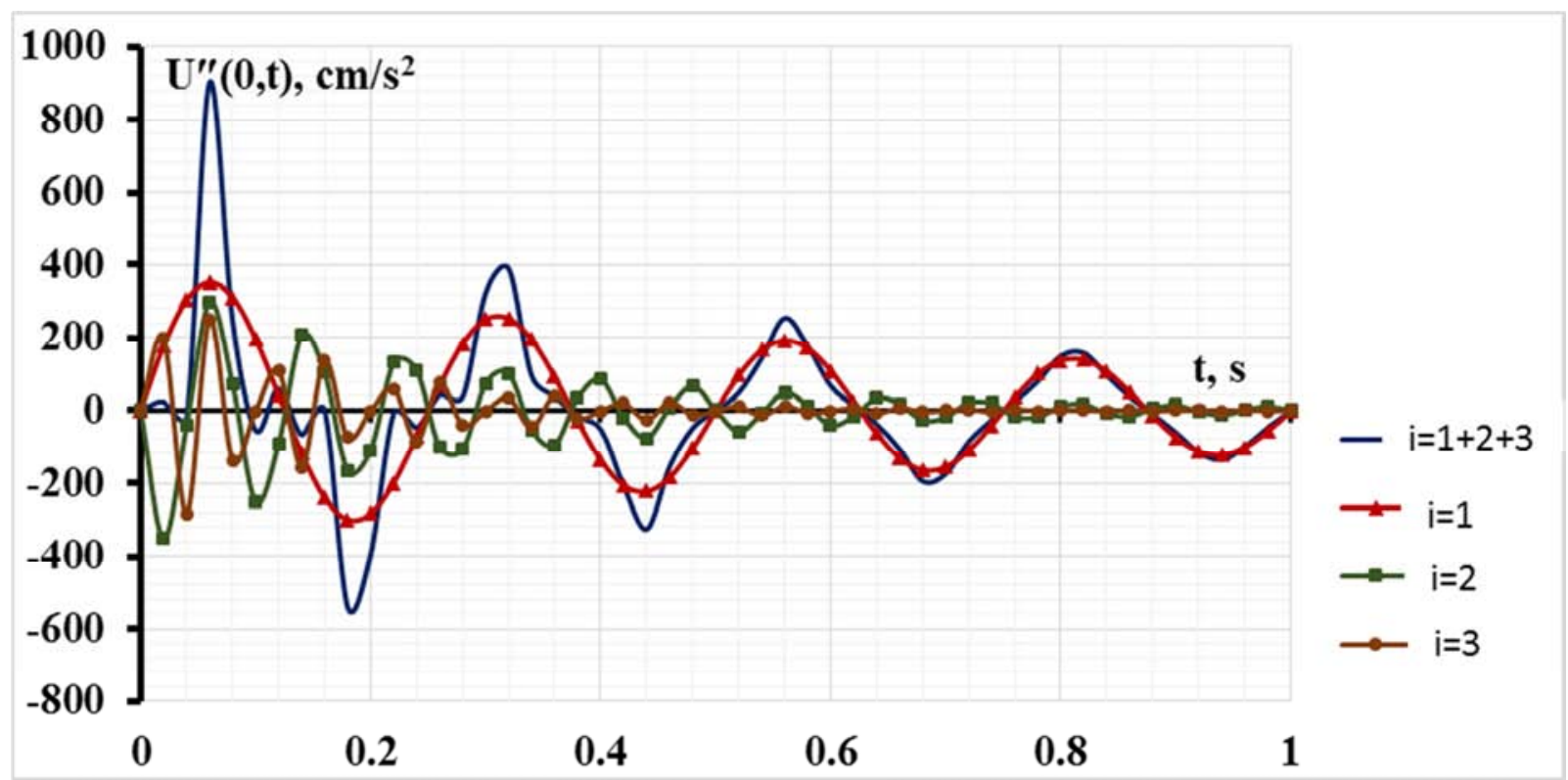

Figure 6. Accelerograms of the ground at $T_{01}=0.25$ s, taking into account the superposition of the first three forms of base vibration according to formula (17) and the first, second and third forms of vibration.

\subsection{Displacements and Accelerations of Soils in the Epicenter Zone}

Table 5 shows the maximum values of displacements and accelerations of solid and loose soils at the rupture $\Delta=0$ and at a distance $\Delta=15 \mathrm{~km}$, from the discontinuity depending on the magnitude of the earthquake $M$. Table 5 shows that the discontinuity (in the epicenter zone) the values of the accelerations of solid soils can already reach one $\mathrm{g}$ at magnitudes $M \geq 7.0$, and with loose soils, only at magnitudes $M \geq 8$.0. At the extreme magnitude of a strong earthquake $M=$ 9.0, the values of accelerations of solid soils can reach up to $8 \mathrm{~g}$, and with loose soils, the accelerations can reach up to $2 \mathrm{~g}$.

Table 5. The maximum values of displacements and accelerations of solid and loose soils at rupture $\Delta=0$ and at a distance $\Delta=15 \mathrm{~km}$, depending on the earthquake magnitude $M$

\begin{tabular}{|c|c|c|c|c|c|c|c|c|}
\hline \multirow[t]{2}{*}{$\begin{array}{l}\text { Earthquake } \\
\text { magnitude, } \\
M\end{array}$} & \multicolumn{2}{|c|}{$\begin{array}{l}\text { Maximal displacement and } \\
\text { acceleration of the ground at the } \\
\text { rupture } \Delta=0 \text { for hard grounds } \\
T_{01}=0.4 \mathrm{~s}\end{array}$} & \multicolumn{2}{|c|}{$\begin{array}{l}\text { Maximal displacement and } \\
\text { acceleration of the ground at the } \\
\text { rupture } \Delta=0 \text { for loose grounds } \\
T_{01}=0.8 \mathrm{~s}\end{array}$} & \multicolumn{2}{|c|}{$\begin{array}{l}\text { Maximal displacement and } \\
\text { acceleration of the ground at } \\
\text { the distance } \Delta=15 \mathrm{~km} \text { for hard } \\
\text { grounds } T_{01}=0.4 \mathrm{~s}\end{array}$} & \multicolumn{2}{|c|}{$\begin{array}{l}\text { Maximal displacement and } \\
\text { acceleration of the ground at } \\
\text { the distance } \Delta=15 \mathrm{~km} \text { for } \\
\text { loose grounds } T_{01}=0.8 \mathrm{~s}\end{array}$} \\
\hline & $U_{\max }, \mathbf{c m}$ & $\begin{array}{l}U^{\prime \prime}{ }_{\text {max }} \text {, in fraction } \\
\text { of } g\end{array}$ & $U_{\max }, \mathbf{c m}$ & $\begin{array}{l}U^{\prime \prime}{ }_{\max } \text {, in fraction } \\
\text { of } \mathrm{g}\end{array}$ & $U_{\max }, \mathbf{c m}$ & $\begin{array}{l}U^{\prime \prime}{ }_{\text {max }} \text {, in fraction } \\
\text { of } g\end{array}$ & $U_{\max }, \mathbf{c m}$ & $\begin{array}{l}U^{\prime \prime}{ }_{\max } \text {, in fraction } \\
\text { of } \mathrm{g}\end{array}$ \\
\hline 6.0 & 0.69 & 0.40 & 1.42 & 0.20 & 0.37 & 0.21 & 0.75 & 0.11 \\
\hline 6.5 & 1.15 & 0.66 & 2.34 & 0.33 & 0.61 & 0.35 & 1.24 & 0.17 \\
\hline 7.5 & 3.11 & 1.79 & 6.37 & 0.89 & 1.65 & 0.95 & 3.38 & 0.47 \\
\hline 8.0 & 5.13 & 2.95 & 10.50 & 1.47 & 2.72 & 1.56 & 5.58 & 0.78 \\
\hline 8.5 & 8.46 & 4.86 & 17.32 & 2.43 & 4.49 & 2.58 & 9.19 & 1.29 \\
\hline 9.0 & 13.95 & 8.01 & 28.55 & 4.01 & 7.41 & 4.25 & 15.16 & 2.13 \\
\hline
\end{tabular}

From Table 4 it is also becomes clear that the values of displacements and accelerations of solid and loose soils at a dispersion of $\Delta=15 \mathrm{~km}$ are approximately half that of the rupture, regardless of the magnitude of the earthquake. With an increase in the magnitude of the earthquake by one unit, the magnitude of displacements and accelerations of soils increase 2.73 times. The displacement and acceleration of soils with an earthquake with a magnitude $M=6.0$ is 20 times smaller than in an earthquake with a magnitude of $\mathrm{M}=9.0$

\subsection{On the Limiting Values of Displacements and Accelerations of Soils}

The maximum displacement and acceleration of the soil, given in the above Table 4, are obtained by an analytical method, assuming the ideal elastic work of the soil. When considering the elastic-plastic stage of the soil work, they will be completely different. At the same time, significant changes, towards the increase, will undergo the values of the maximum displacement $\boldsymbol{U}_{\max }$ of the soil with the prevalence of the residual deformations.

For example, according to the average statistical empirical 
estimates at $M=7.0$ in the focal zone, with general PGD = $40 \mathrm{~cm}$ soil displacements, the residual displacement for rocky soils is $37 \mathrm{~cm}$, therefor, the elastic displacement will be 40-37 $=3 \mathrm{~cm}$, and for loose soils with PGD $=145 \mathrm{~cm}$, the residual displacement is $140 \mathrm{~cm}$, consequently the elastic displacement will be $145-140=5 \mathrm{~cm}$ [16]. Below is a small calculation showing that for rocks the beginning of destruction (the end of elastic deformation) can occur in displacements within 0.6-5 $\mathrm{cm}$. In an elastic medium from a spreading transverse wave

$$
U(t, \xi)=f\left(t-\frac{\xi}{\mathrm{v}_{s}}\right),
$$

at a distance $\xi$ at time t s a certain relative shear deformation and velocity of soil particles, the values of which will be:

$$
\frac{\partial U}{\partial \xi}=-\frac{1}{\mathrm{v}_{s}} f^{\prime}\left(t-\frac{\xi}{\mathrm{v}_{\mathrm{s}}}\right), \frac{\partial U}{\partial t}=\mathrm{v}_{g}=f^{\prime}\left(t-\frac{\xi}{\mathrm{v}_{s}}\right),
$$

In case of a plane transverse wave, as can be seen from (41), for the relative shear deformation $\gamma$, we have

$$
\gamma=\frac{\partial U}{\partial \xi}=-\frac{1}{\mathrm{v}_{s}} \frac{\partial U}{\partial t}=\frac{\mathrm{v}_{g}}{\mathrm{v}_{s}}, \quad \mathrm{v}_{g}=\gamma \mathrm{v}_{s}
$$

Therefore, the process of determining the value of deformation $\gamma$ is essentially simplified, since this can be done indirectly using the value of the oscillation velocity, that is instead of a complex operation of setting up the difference $\Delta U=U_{2}-U_{1}$ of the displacement of two points of the medium, one can start from the earthquake velisogram $\partial U / \partial t$ at one point of the soil and the magnitude of the wave propagation velocity $v_{s}$. For a harmonic plane transverse wave, we have

$$
U(t, \xi)=U_{\max } \cos \frac{2 \pi}{T_{0}}\left(t-\frac{\xi}{\mathrm{v}_{s}}\right), \mathrm{v}_{g}=\frac{\partial U}{\partial t}=U_{\max } \frac{2 \pi}{T_{0}}
$$

where $U_{\max }$ is the amplitude of soil vibration, $T_{0}$ is the period of oscillation of the soil particles, $v_{s}$ is the transverse waves propagation velocity. On the basis of (41) and (42), in this case we will have

$$
\gamma=\frac{U_{\max } 2 \pi}{\mathrm{v}_{s} T_{0}} .
$$

The rocks of the earth's crust can withstand a certain shear deformation, after which shear cracks are formed in the medium. If denote the limiting shear deformation of the medium through $\gamma_{\lim }$, then from (44) the soil vibration amplitude $U_{\max }$, is

$$
U_{\max }=\frac{\mathrm{v}_{s}}{2 \pi} T_{0} \gamma_{\lim } .
$$

Similarly, for the soil particles oscillation velocity $\mathrm{v}_{g}=U^{\prime}$ and acceleration of soil $U^{\prime \prime}$ under elastic vibrations, we have

$$
U_{\max }^{\prime}=\mathrm{v}_{s} \gamma_{\lim }, \quad U_{\max }^{\prime \prime}=\mathrm{v}_{s} \frac{2 \pi}{T_{0}} \gamma_{\lim }
$$

The magnitude $\gamma_{\lim }=(0.5 \div 2.0) \times 10^{-4}$ is considered the most probable for most earthquakes [6, 12, 24, 26]. According to the results of laboratory tests of soil samples, it is believed that $\gamma_{\lim }$ of the rocks of the earth's crust can reach the value $\gamma_{\lim }=10^{-3}$ [26]. For sixteen variants of rocky soils, the values of maximum displacement $U_{\max }$, the velocity $U_{\max }^{\prime}$ of oscillation of soil particles and the maximum acceleration $U_{\max }^{\prime \prime}$ of the soil, calculated by (45) and (46), are listed in Table 6 .

Table 6. The values of the maximum elastic displacements, velocities and accelerations of rocky soils.

\begin{tabular}{lllllll}
\hline $\begin{array}{l}\text { Options } \\
\text { of soils }\end{array}$ & $\gamma_{\text {lim }}$ & $\begin{array}{c}\mathrm{V}_{\mathrm{s}}, \\
\mathbf{m} / \mathbf{s}\end{array}$ & $\begin{array}{l}\boldsymbol{T}_{\mathbf{0 1}}, \\
\mathbf{s}\end{array}$ & $\begin{array}{c}\boldsymbol{U}_{\max }, \\
\mathbf{c m}\end{array}$ & $\begin{array}{c}\boldsymbol{U}_{\max }^{\prime}, \\
\mathbf{c m} / \mathbf{s}\end{array}$ & $\begin{array}{c}\boldsymbol{U}_{\text {max }}^{\prime \prime} \\
\mathbf{c m} / \mathbf{s}^{\mathbf{2}}\end{array}$ \\
\hline 1 & $10^{-3}$ & 1500 & 0.2 & 4.77 & 150.0 & 4710 \\
2 & $0.5 \cdot 10^{-3}$ & 1200 & 0.3 & 2.86 & 60.0 & 1300 \\
3 & $2 \cdot 10^{-4}$ & 1000 & 0.4 & 1.26 & 20.0 & 320 \\
4 & $1 \cdot 10^{-4}$ & 800 & 0.5 & 0.63 & 8.0 & 100 \\
5 & $1.5 \cdot 10^{-4}$ & 3000 & 0.1 & 0.72 & 45.0 & 2826 \\
6 & $1.25 \cdot 10^{-4}$ & 2500 & 0.15 & 0.75 & 37.25 & 1309 \\
7 & $1 \cdot 10^{-4}$ & 2000 & 0.20 & 0.64 & 20.0 & 628 \\
8 & $0.8 \cdot 10^{-4}$ & 1500 & 0.25 & 0.48 & 12.0 & 301 \\
9 & $0.6 \cdot 10^{-4}$ & 1200 & 0.30 & 0.34 & 7.0 & 150 \\
10 & $0.5 \cdot 10^{-4}$ & 1000 & 0.35 & 0.27 & 5.0 & 90 \\
11 & $0.5 \cdot 10^{-3}$ & 1500 & 0.08 & 0.95 & 75.0 & 5800 \\
12 & $0.5 \cdot 10^{-3}$ & 1200 & 0.1 & 0.95 & 60.0 & 3760 \\
13 & $2 \cdot 10^{-4}$ & 1000 & 0.12 & 0.38 & 20.0 & 1050 \\
14 & $1.5 \cdot 10^{-4}$ & 900 & 0.13 & 0.28 & 14.0 & 650 \\
15 & $1.25 \cdot 10^{-4}$ & 800 & 0.15 & 0.24 & 10.0 & 420 \\
16 & $1 \cdot 10^{-4}$ & 700 & 0.17 & 0.19 & 7.0 & 260 \\
\hline
\end{tabular}

By the way, according to the same E. F. Savorensky [18] methodology, for granite at $\mathrm{v}_{\mathrm{s}}=3300 \mathrm{~cm} /$ сек and $\mathrm{T} 01=$ $0.25 \mathrm{~s}$ the variation range of $U_{\max }$ is within $1 \mathrm{~cm}$ to $10 \mathrm{~cm}$. When taking into account the damping of waves, due to internal friction in rocks, the values $U_{\max }, U_{\max }^{\prime}, U_{\max }^{\prime \prime}$ presented in Table 6 can be reduced by approximately $20-25 \%$. Thus, in the case of elastic oscillations of rocky soils, the maximum values of displacements, velocities, and accelerations of the rocky soil can reach, respectively, up to $3.57 \mathrm{~cm}, 112 \mathrm{~cm} / \mathrm{s}$, and $2128 \mathrm{~cm} / \mathrm{s}^{2}$ without their destruction.

Occurrence of high soil accelerations on the earth surface is apparently are conditioned not by the values of the period of the main form of oscillations of the soil section $T_{01}$, but to the periods of the second $T_{02}$ or third waveform $T_{03}$ (32), which are respectively 3 and 5 times smaller than the period of the first waveform. This is evidenced by the values of maximum displacements, velocities, and accelerations presented in Table 6 , corresponding to the variants of soils 11-16, for which the values of the periods $T_{01}$ have been calculated according to the traditional formula $T_{01}=4 H / \mathrm{v}_{s}$, with the layer thickness $\mathrm{H}=30 \mathrm{~m}$. 
The amplitudes of soil displacements along surface waves in strong earthquakes propagating at large distances reach up to $3 \mathrm{~cm}$ with a period $\mathrm{T}_{0}=20 \mathrm{sec}$, but they do not represent any danger [25].

Such estimates for earth (loose) soils are unacceptable, since the process of discontinuity disruption in such soils is of more complex nature. They during the strong earthquakes are subjected to either liquefaction, or uneven precipitation, which can reach several meters. These phenomena pose a serious danger to buildings and structures (they can fall without destruction), if they are calculated even for high horizontal acceleration of the ground.

Table 7. Frequencies of harmonic oscillations (in hertz), corresponding to the given displacements $U$ (cm) and the soil accelerations $U^{\prime \prime}$ (in fractions of g).

\begin{tabular}{|c|c|c|c|c|c|c|}
\hline \multirow{2}{*}{$U^{\prime \prime}$ in fractions of $g$} & \multicolumn{6}{|l|}{$U, \mathrm{~cm}$} \\
\hline & 0.0001 & 0.001 & 0.01 & 0.1 & 1.0 & 10.0 \\
\hline 1.0 & 500 & 160 & 50 & 16 & 5 & 1.6 \\
\hline 0.5 & 356 & 113 & 36 & 11 & 3.6 & 1.1 \\
\hline 0.1 & 160 & 50 & 16 & 5 & 1.6 & 0.5 \\
\hline 0.01 & 50 & 16 & 5 & 1.6 & 0.5 & 0.16 \\
\hline
\end{tabular}

Fat numbers indicate the fluctuations expected in case of earthquakes of moderate strength.

In conclusion, we note that we share the opinion of $\mathrm{Ch}$. Richter [25] that waves with maximum displacements of the soil do not coincide with the waves of maximum ground accelerations. Large values of ground accelerations are associated with small ground displacements, and large soil displacements are associated with low frequencies and low soil accelerations. A graphic illustration of this is provided by the data given in Table. 7, borrowed from the work of $\mathrm{Ch}$. Richter [25], with some additions developed by the author.

\section{Main Results}

We developed a method of forecasting the acceleration and displacement values during strong soil dis placements induced by an earthquake considering it as an instantaneous rupture of the Earth's surface. This method is based on the fact that near surface rock layers have a much greater degree of horizontal shear deformation compared to deeper layers. Therefore, for instantaneous rupture of the Earth's crust an influence of inertial motions of deep rock layers on that of the surface layers can be neglected. According to this, the heterogeneous soil stratum of a thickness $H$ to the bed rock with $\mathrm{v}_{S}>1000 \mathrm{~m} / \mathrm{sec}$, all layers of which have the velocity $v$ at the beginning of an earthquake, are used as a scheme for the calculation of the regularities of variations displacements (seismogram) of soil particles on the surface over time. Having analyzed the data in $[1,4,6,11,15$, $22]$, we propose to determine an initial velocity value $\mathbf{v}$ as the function of a predicted earth quake magnitude $M$ and a distance $\Delta$ from the rupture to the observation point by formula (3) and the data given in Table 1.

Using the solution of the wave equations of trans verse vibrations in layers (6) with the boundary and initial conditions (7), (8), and (9), the analytical expressions (28) of displacements and accelerations for all levels of $n$ layered geological section were obtained. Their main feature is that for a given predicted earthquake magnitude $M$ and a distance $\Delta$ from the observation point (a construction site) to a predicted rupture (active fault), one can establish not only the maximum soil displacement and acceleration values based on the local soil conditions, and their variations over time, but also the values of the predominant vibration periods in the soil.

The results obtained are illustrated by examples of a homogenous surface layer with solid or loose soils at $M=7$ and $\Delta=15 \mathrm{~km}$ (Figures 4 and 5). The proposed method can be used for the development of maps of seismic zoning and seismic hazard assessment of construction sites of especially responsible individual objects.

\section{Conclusion}

1. It is shown that the not instantaneous rupture leads to an insignificant decrease in the initial rate of oscillation of the soil particles and, consequently, to the same mitigation of the earthquake effect on the ground surface. The values of the rates and intensities of earthquakes, established by the developed method, have been compared with the intensities established on the basis of the MSK-64 seismic scale, showing a sufficient correlation for earthquakes with $\mathrm{V}-\mathrm{X}$ intensity and magnitudes $6.0 \leq \mathrm{M} \leq 8.0$ (Figure3). Dependences of $\mathrm{v}(\Delta)$ for values of magnitudes $6.0 \leq \mathrm{M} \leq 9.0$ and earthquake intensity on the MSK-64 scale were plotted, which, by analogy with dependences of the decrease of the maximum value of the soil acceleration as a function of the epicentral distance, can be called the earthquake intensity attenuation curves (Figure 3 ).

2. Figures 4, 5 and Table 4 show synthetic seismograms and accelerograms plotted according to the basic formula (38) for various soil bases of seismic properties (Table 3). The duration of intensive vibrations on loose soils is up to 3 times greater than on hard soils. Taking into account the higher forms of oscillation of the base (the second and third forms) for hard and loose soils leads to an increase in the maximum accelerations of soils, calculated only for the first form of oscillation, 2.53 times on average, and only 1.1 times for maximum soil displacements.

3. The values of hard soils accelerations can already reach $1 \mathrm{~g}$ at $\mathrm{M} \geq 7.0$ magnitude, and with loose soils only at $\mathrm{M} \geq 8.0$ magnitude. With an increase of the earthquake magnitude by one whole unit, the displacement and acceleration of soils increase 2.73 times. The displacement and acceleration in an earthquake with a magnitude $\mathrm{M}=6.0$ is 20 times smaller than for an 
earthquake of $\mathrm{M}=9.0$. The values of displacements and accelerations of hard and loose soils at a distance of $\Delta=$ $15 \mathrm{~km}$ from the rupture are about half that of the rupture, regardless of the magnitude of the predicted earthquake.

4. In presents developed method determining the limiting values displacements, velocities and accelerations for rocky soils, assuming the ideal elastic work of the soil (Table 6).

\section{References}

[1] J. N. Brune, The physics of earthquake strong motion, in Seismic Risk and Engineering Decisions, Lomnitz, C. and Rosenblueth, Editors, New York: Elsevier, 1976, pp. 141-177.

[2] G. Butcher, D. Hopkins, R. Jury, W. Massey, G. McKay, and G. McVerr, The September 1985 Mexico earthquakes: Final Report of the New Zealand Reconnaissance Team, Bull. N. Z. Soc. Earthquake Eng., 1988, vol. 21, no. 1.

[3] H. Jeffreys, and S B. Wirles, Methods of Mathematical Physics, Cambridge: Cambridge Univ., 1950, 2nd ed.

[4] L. Esteva, Seismicity, in Seismic Risk and Engineering Deci sions, Lomnitz, C. and Rosenblueth, Editors, New York: Elsevier, 1976, pp. 179-224.

[5] E. Faccioli, and D. Resendiz, Soil dynamics: Behavior including liquefaction, in Seismic Risk and Engineering Decisions, Lomnitz, C. and Rosenblueth, Editors., New York: Elsevier, 1976, pp. 71-140.

[6] K. Kasahara, Earthquake Mechanics, Cambridge: Cambridge University, Press, 1981.

[7] Khachiyan E. Y. On Basic Concepts for Development of United International Earthquake Resistant Construction Code. Earthquake Hazard and Seismic Risk Reduction. Editors S. Balassanian, A. Cisternas and M. Melkumyan, Kluwer Academic Publishers, Netherlands, 2000, pp. 333-343.

[8] N. M. Newmark and E. Rosenblueth Fundamentals of Earthquake Engineering. Prentice-Hall, Inc. EnglewoodCliffs, N. Y.

[9] E. Y. Khachiyan A Method of Determination of Dominant Vibration Periods Values for Nonhomogeneous Multilayer Ground Sites. Horizon Research Publishing Corporation, USA Universal Journal of Engineering Science 2013, vol. 1(3), pp 57-67.

[10] E. E. Khachiyan, Prikladnaya seismologiya (Applied Seis mology), Yerevan: Gitutyun, 2008. (in Russian).

[11] E. Y. Khachyian On a Simple Method for Determining the Potential Strain Energy Stored in the Earth before a Large Earthquake. ISSN 0742-0463, Journal of Volcanology and Seismology, 2011, Vol. 5, No. 4, pp. 286-297. Pleiades Publishing, Ltd., 2011.

[12] E. E. Khachiyan, On Determining of the Ultimate Strain of
Earth Crust Rocks by the Value of Relative Slips on the Earth Surface after a Large Earthquake. Science Publishing Group, Earth Sciences, doi: 10. 11648/j.earth. 20160506. 14 ISSN: 2328-5974 (Print); ISSN: 2328-5982 (Online) Received: October 26, 2016; Accepted: November 10, 2016; Published: December 21, 2016 Vol. 5, No. 6 pp 111-118, http://www.sciencepublishinggroup.com/j/earth.

[13] E. E. Khachiyan, Method for Determining the Potential Strain Energy Stored in the Earth before a Large Earthquake. Science Publishing Group, USA Earth Science, vol. 2, 2, 2013 pp 47-57

[14] E. Y. Khachiyan On the Possibility of Predicting Seismogram and Accelerogram of Strong Motions of the Soil for an Earthquake Model Considered as an Instantaneous Rupture of the Earth's Surface. ISSN 0747 9239, Seismic Instruments, 2015, Vol. 51, No. 2, pp. 129-140. (C) Allerton Press, Inc., 2015.

[15] C. Lomnitz, and K. S. Singh, Earthquakes and earthquake prediction, in Seismic Risk and Engineering Decisions, Lomnitz, C. and Rosenblueth, Editors., New York: Elsevier, 1976, pp. 3-30.

[16] N. N. Mikhailova, and F. F. Aptikaev, Some correlation relations between parameters of seismic motions, J. Earth quake Pred. Res., 1996, vol. 5, no. 5, pp. 257-267.

[17] Okamoto S. Introduation to Earthquake Engineering. University of Tokyo Press, 1973.

[18] E. F. Savarenskii, Seismicheskie volny (Seismic Waves), Moscow: Nedra, 1972. (in Russian)

[19] SNRA II6. 02. 2006. Seismostoikoe stroitel'stvo: Normy proektirovaniya (Construction Regulations of Republic of Armenia II6. 02. 2006. EarthquakeResistant Building: Design Standards), Yerevan, 2006.

[20] L. R. Stavnitser, Seismostoikost' osnovanii i fundamentov (Seismic Resistance of Foundations), Moscow: Izd. Assots. stroit. vuzov, 2010. (in Russian)

[21] A. N. Tikhonov, and A. A. Samarskii, Uravneniya matemat icheskoi fiziki (Equations of Mathematical Physics), Mos cow: Nauka, 1977. (in Russian)

[22] D. L. Wells, and K. I. Coppersmith, New empirical rela tionship among magnitude, rupture length, rupture width, rupture area, and surface displacement, Bull. Seismol. Soc. Am., 1994, vol. 84, no. 4, pp. 974-1002.

[23] V. Zelenovich, and T. Paskalev, Yugoslav code for aseismic design and analysis of engineering structures in seismic regions, Proceedings of the 8th European Conference of Earthquake Engineering, Lisbon, 1986, vol. 1, pp. 361-369.

[24] Rikitake T. Earthquake Predction. Elsevier Scientific Publishing. CoAmsterdam, 1976, 357p.

[25] Ch. F. Richter Elementary Seismology. W. H. Freeman and Co., San Francisco, 1958, 768p.

[26] Mogi K. Earthquake Prediction Academic Press, 1985. 